TRANSACTIONS OF THE

AMERICAN MATHEMATICAL SOCIETY

Volume 362, Number 2, February 2010, Pages 753-776

S 0002-9947(09)04946-0

Article electronically published on September 14, 2009

\title{
ON THE CLUSTER MULTIPLICATION THEOREM FOR ACYCLIC CLUSTER ALGEBRAS
}

\author{
FAN XU
}

\begin{abstract}
Caldero and Keller, and Hubery have proved the cluster multiplication theorems for finite type and affine type. We generalize their results and prove the cluster multiplication theorem for arbitrary type using the 2Calabi-Yau property and a property we call 'higher order associativity'.
\end{abstract}

\section{INTRODUCTION}

Cluster algebras were introduced by Fomin and Zelevinsky in [9]. By definition, cluster algebras are commutative algebras generated by a set of variables called cluster variables. Let $Q$ be a quiver. We denote by $\mathcal{A}(Q)$ the associated cluster algebra. If $Q$ does not contain oriented cycles, we call $Q$ an acyclic quiver. The cluster algebras associated to acyclic quivers are called acyclic cluster algebras. Their relations to quiver representations were first revealed in [20. In [1, the authors found a general framework for the link of cluster algebras and quiver representations. They introduced the cluster categories as the categorification of acyclic cluster algebras. For an acyclic quiver $Q$, the associated cluster category $\mathcal{C}(Q)$ is the orbit category of the bounded derived category $\mathcal{D}^{b}(\bmod k Q)$ over a field $k$ by the auto-equivalence $F:=[1] \tau^{-1}$, where [1] is the translation functor and $\tau$ is the Auslander-Reiten translation. In general, one can define the cluster category of a hereditary category with Serre duality $\nu$ by taking $\tau=[-1] \nu$ as shown in [17].

In 2, the authors introduced a certain structure of Hall algebra involving the cluster category $\mathcal{C}(Q)$ by associating to the objects in $\mathcal{C}(Q)$ some variables given by an explicit map $X_{\text {? }}$, called the Caldero-Chapoton map. We denote by $X_{M}$ the variable (called the generalized cluster variable) associated to an object $M$ in $\mathcal{C}(Q)$. In the case where $M$ is a non-projective $k Q$-module, the authors gave the multiplication of $X_{M}$ and $X_{\tau M}$ as follows:

$$
X_{\tau M} X_{M}=X_{B}+1,
$$

where $B$ is the middle term of the almost split sequence involving $M$ and $\tau M$.

If $Q$ is a simply laced Dynkin quiver, Caldero and Keller [3] extended the above multiplication (0.1) to the multiplication of any two variables associated to two

Received by the editors November 15, 2007.

2000 Mathematics Subject Classification. Primary 16G20, 16G70; Secondary 14M99, 18E30.

Key words and phrases. 2-Calabi-Yau, cluster category.

This research was partially supported by the NSF of China (No. 10631010).

(C)2009 American Mathematical Society 
objects in $\mathcal{C}(Q)$ as follows:

$$
\chi_{c}\left(\mathbb{P} \operatorname{Ext}^{1}(M, N)\right) X_{M} X_{N}=\sum_{Y}\left(\chi_{c}\left(\mathbb{P E x t}^{1}(M, N)_{Y}\right)+\chi_{c}\left(\operatorname{PExt}^{1}(N, M)_{Y}\right)\right) X_{Y},
$$

where $\chi_{c}$ is the Euler-Poincaré characteristic of étale cohomology with proper support, $M, N \in \mathcal{C}(Q)$ and $Y$ runs through the isomorphism classes of $\mathcal{C}(Q)$. This is called the cluster multiplication theorem for finite type.

The above cluster multiplication theorem was generalized to affine type in [14] by using Green's theorem and the existence of Hall polynomials for affine quivers. A cluster multiplication theorem for indecomposable regular modules over the path algebra of an affine quiver was proved in 7]. In 21, the author gave the cluster multiplication theorem in the case when $\operatorname{dim}_{k} \operatorname{Ext}^{1}(M, N)=1$ and introduced the cluster character for an arbitrary 2-Calabi-Yau category with cluster-tilting objects.

The aim of this paper is to generalize the cluster multiplication theorems for finite and affine types to arbitrary type. Note that there is an alternative proof of the cluster multiplication theorem for arbitrary type in 31] by applying the projective version of Green's theorem under the action of $\mathbb{C}^{*}$. Compared with [31, the present proof has the following differences. First, it is more direct and simpler. The present proof is independent of the projective version of Green's formula and only involves the 2-Calabi-Yau property (in the guise of the AuslanderReiten formula) and a property we call 'higher order associativity' and which is analogous to the associativity of the multiplication of a derived Hall algebra defined in 29]; see Section 3.2 for details. Second, it is more accessible. The present proof uses Euler characteristics of algebraic varieties instead of quasi-Euler characteristics of orbit spaces of algebraic varieties under the actions of algebraic groups in [31. Third, it holds greater promise in view of a future generalization to hereditary categories more general than module categories of hereditary algebras.

The interaction between cluster algebras and the representation theory of a quiver naturally leads to the question whether there are cluster algebras associated to the cluster categories of the categories of coherent sheaves over weighted projective lines or elliptic curves. Also, it is meaningful to ask what is the corresponding cluster multiplication theorem. The intuitive idea is to extend the method in 31. However, the proof in [31] heavily depends on Green's theorem. Also, the proof of the projective version of Green's formula in [31] is complicated. We need to look for a new approach not involving Green's theorem.

From the combinatorial point of view, the higher order associativity in the present proof is analogous to the associativity of the multiplication in derived Hall algebras under combinatorial context. Hence, we can hope that the property of higher order associativity holds for categories of coherent sheaves over weighted projective lines or elliptic curves if we put the property into a suitable geometric context (see Remark 4.2). The situation is similar for the 2-Calabi-Yau property.

This paper is organized as follows. In Section 1, we recall the general theory involving the computation of Euler characteristics of algebraic varieties and the cluster category needed in this paper. In order to use the proposition in Section 1 to compute Euler characteristics, we need to construct some morphisms of varieties. Section 2 is devoted to this aim. In Section 3, we prove an equation called the higher order associativity. The cluster multiplication theorem for arbitrary type is stated and proved in the last section. As an application of the proof of the main theorem, 
we induce the formula (0.1). Finally, we illustrate our theorem through an example which has been studied in detail in [5] and [33].

\section{Preliminaries}

Let $Q=\left(Q_{0}, Q_{1}, s, t\right)$ be a finite acyclic quiver where $Q_{0}$ and $Q_{1}$ are the finite sets of vertices and arrows, respectively, and $s, t: Q_{1} \rightarrow Q_{0}$ are maps such that any arrow $\alpha$ starts at $s(\alpha)$ and terminates at $t(\alpha)$. A dimension vector $\mathbf{d}$ for $Q$ is a function from $Q_{0}$ to $\mathbb{N}$. We write $d_{i}$ instead of $\mathbf{d}(i)$ for any $i \in Q_{0}$. Let $\mathbb{C} Q$ be the path algebra of $Q$ over $\mathbb{C}$. We denote by $\bmod \mathbb{C} Q$ the category of finite-dimensional $\mathbb{C} Q$-modules.

1.1. Euler characteristics and the pushforward functor. For any dimension vector $\mathbf{d}=\left(d_{i}\right)_{i \in Q_{0}}$, we consider the affine space over $\mathbb{C}$

$$
\mathbb{E}_{\mathbf{d}}=\mathbb{E}_{\mathbf{d}}(Q)=\bigoplus_{\alpha \in Q_{1}} \operatorname{Hom}_{\mathbb{C}}\left(\mathbb{C}^{d_{s(\alpha)}}, \mathbb{C}^{d_{t(\alpha)}}\right) .
$$

Any element $x=\left(x_{\alpha}\right)_{\alpha \in Q_{1}}$ in $\mathbb{E}_{\mathbf{d}}$ defines a representation $M(x)=\left(\mathbb{C}^{\mathbf{d}}, x\right)$ where $\mathbb{C}^{\mathbf{d}}=\bigoplus_{i \in Q_{0}} \mathbb{C}^{d_{i}}$. Naturally we can define the action of the algebraic group $G_{\mathbf{d}}(Q)=\prod_{i \in Q_{0}} G L\left(\mathbb{C}^{d_{i}}\right)$ on $\mathbb{E}_{\mathbf{d}}$ by $g \cdot x=\left(g_{t(\alpha)} x_{\alpha} g_{s(\alpha)}^{-1}\right)_{\alpha \in Q_{1}}$.

Let $X$ be an algebraic variety over $\mathbb{C}$. A constructible function $f: X \rightarrow \mathbb{Q}$ satisfies that $f(X)$ is a finite subset of $\mathbb{Q}$ and $f^{-1}(c)$ is a constructible subset of $X$ for any $c \in \mathbb{Q}$. Write $M(X)$ for the $\mathbb{Q}$-vector space of constructible functions on $X$. Now, suppose $\mathcal{O}$ is a constructible subset of $\mathbb{E}_{\mathbf{d}}$. The function $1_{\mathcal{O}}$ is called a characteristic function if $1_{\mathcal{O}}(x)=1$ for any $x \in \mathcal{O}$, and 0 otherwise. It is clear that $1_{\mathcal{O}}$ is the simplest constructible function and any constructible function is a linear combination of characteristic functions. We say $\mathcal{O}$ is $G_{\mathbf{d}}$-invariant if $G_{\mathbf{d}} \cdot \mathcal{O}=\mathcal{O}$. In this case, $1_{\mathcal{O}}$ is called a $G_{\mathbf{d}}$-invariant.

In the following, the constructible sets and functions will always be assumed $G_{\mathbf{d}}$-invariant unless specifically mentioned.

Let $\chi$ denote the Euler characteristic in compactly-supported cohomology. Let $X$ be an algebraic variety and $\mathcal{O}$ a constructible subset of $X$ which is the disjoint union of finitely many locally closed subsets $X_{i}$ for $i=1, \cdots, m$. Define $\chi(\mathcal{O})=$ $\sum_{i=1}^{m} \chi\left(X_{i}\right)$. We note that it is well-defined. We have the following properties of $\chi$.

Proposition 1.1 (23] and [16]). Let $X, Y$ be algebraic varieties over $\mathbb{C}$. Then

(1) If the algebraic variety $X$ is the disjoint union of finitely many constructible sets $X_{1}, \cdots, X_{r}$, then

$$
\chi(X)=\sum_{i=1}^{r} \chi\left(X_{i}\right)
$$

(2) If $\varphi: X \longrightarrow Y$ is a morphism such that all fibers have the same Euler characteristic $\chi$, then $\chi(X)=\chi \cdot \chi(Y)$.

(3) $\chi\left(\mathbb{C}^{n}\right)=1$ and $\chi\left(\mathbb{P}^{n}\right)=n+1$ for all $n \geq 0$.

We recall the pushforward functor from the category of algebraic varieties over $\mathbb{C}$ to the category of $\mathbb{Q}$-vector spaces (see [19] and [16]). Let $\phi: X \rightarrow Y$ be a morphism of varieties. For $f \in M(X)$ and $y \in Y$, define

$$
\phi_{*}(f)(y)=\sum_{c \neq 0} c \chi\left(f^{-1}(c) \cap \phi^{-1}(y)\right) .
$$


Theorem 1.2 ([6, 16]). Let $X, Y$ and $Z$ be algebraic varieties over $\mathbb{C}, \phi: X \rightarrow Y$ and $\psi: Y \rightarrow Z$ be morphisms of varieties, and let $f \in M(X)$. Then $\phi_{*}(f)$ is constructible, $\phi_{*}: M(X) \rightarrow M(Y)$ is a $\mathbb{Q}$-linear map and $(\psi \phi)_{*}=\psi_{*} \phi_{*}$ is a $\mathbb{Q}$-linear map from $M(X)$ to $M(Z)$.

Given a $\mathbb{C} Q$-module $M$ and any dimension vector $\mathbf{e} \in \mathbb{N}^{Q_{0}}$, we denote by $\operatorname{Gr}_{\mathbf{e}}(M)$ the set of submodules $M_{1} \subset M$ such that $\operatorname{dim} M_{1}=\mathbf{e}$. It is a closed subvariety of the product of Grassmannians of subspaces $\prod_{i \in Q_{0}} \mathrm{Gr}_{e_{i}}\left(\mathbb{C}^{d_{i}}\right)$. Here, $\operatorname{dim} M=\mathbf{d}$. Set

$$
\operatorname{Gr}_{\mathbf{e}}\left(\mathbb{E}_{\mathbf{d}}\right)=\left\{\left(M, M_{1}\right) \mid M \in \mathbb{E}_{\mathbf{d}}, M_{1} \in \operatorname{Gr}_{\mathbf{e}}(M)\right\} .
$$

Proposition 1.3. Let $\mathbf{d}$ and $\mathbf{e}$ be two dimension vectors. Then the function $f$ : $\mathbb{E}_{\mathbf{d}} \rightarrow \mathbb{Q}$ sending $M$ to $\chi\left(\operatorname{Gr}_{\mathbf{e}}(M)\right)$ is a $G_{\mathbf{d}}$-invariant constructible function.

Proof. Consider the natural projection $\phi: \mathrm{Gr}_{\mathbf{e}}\left(\mathbb{E}_{\mathbf{d}}\right) \rightarrow \mathbb{E}_{\mathbf{d}}$. The map $\phi$ is algebraic. By Theorem 1.2 we know that $\phi_{*}\left(1_{\mathrm{Gr}_{\mathbf{e}}\left(\mathbb{E}_{\mathbf{d}}\right)}\right)=f$ is constructible.

For fixed $\mathbf{d}$, there are only finitely many choices of $\mathbf{e}$ such that $\mathrm{Gr}_{\mathbf{e}}\left(\mathbb{E}_{\mathbf{d}}\right)$ is nonempty. For $M \in \mathbb{E}_{\mathbf{d}}$, we define [12, Section 1.2]

$$
\langle M\rangle:=\left\{M^{\prime} \in \mathbb{E}_{\mathbf{d}} \mid \chi\left(\operatorname{Gr}_{\mathbf{e}}\left(M^{\prime}\right)\right)=\chi\left(\operatorname{Gr}_{\mathbf{e}}(M)\right) \text { for any } \mathbf{e}\right\} .
$$

Proposition 1.3 has the following corollary.

Corollary 1.4. There exists a finite subset $S(\mathbf{d})$ of $\mathbb{E}_{\mathbf{d}}$ such that

$$
\mathbb{E}_{\mathbf{d}}=\bigsqcup_{M \in S(\mathbf{d})}\langle M\rangle
$$

1.2. The cluster category. Given an acyclic quiver $Q$ and $i \in Q_{0}$, we denote by $S_{i}$ the simple $\mathbb{C} Q$-module associated to $i$, by $P_{i}$ its projective cover and by $I_{i}$ its injective hull. Given a $\mathbb{C} Q$-module $M$, we denote by $\operatorname{dim} M$ its dimension vector. For any $i \in Q_{0}$, we will always denote by $s_{i}$ the $i$-th vector of the canonical basis of $\mathbb{Z}^{Q_{0}}$. In particular, for any $i \in Q_{0}$ we have $\operatorname{dim} S_{i}=s_{i}$. We denote by $\langle-,-\rangle$ the Euler form on $\mathbb{C} Q$-mod given by

$$
\langle M, N\rangle:=\langle\operatorname{dim} M, \operatorname{dim} N\rangle=\operatorname{dim}_{\mathbb{C}} \operatorname{Hom}_{\mathbb{C Q}}(M, N)-\operatorname{dim}_{\mathbb{C}} \operatorname{Ext}_{\mathbb{C} Q}^{1}(M, N)
$$

for any $\mathbb{C} Q$-modules $M$ and $N$. In the following, for any additive category $\mathcal{F}$, we denote by ind $(\mathcal{F})$ the subcategory of $\mathcal{F}$ formed by a system of representatives of the isomorphism classes of indecomposable objects in $\mathcal{F}$.

Let $\mathcal{D}^{b}(Q)$ be the bounded derived category of $\bmod \mathbb{C} Q$ with the shift functor [1] and the AR-translation $\tau$. The cluster category associated to $Q$ is the orbit category $\mathcal{C}=\mathcal{C}(Q):=\mathcal{D}^{b}(Q) / F$ with $F=[1] \tau^{-1}$. It is proved in [17 that $\mathcal{C}$ is a triangulated category with the canonical triangle functor $\mathcal{D}^{b}(Q) \rightarrow \mathcal{C}$. As in [1] and 3], the category $\mathcal{C}$ is 2-Calabi-Yau, i.e., there is an almost canonical non-degenerate bifunctorial pairing

$$
\phi: \operatorname{Ext}_{\mathcal{C}}^{1}(M, N) \times \operatorname{Ext}_{\mathcal{C}}^{1}(N, M) \rightarrow \mathbb{C} .
$$

Here, the 2-Calabi-Yau property can be deduced from the Auslander-Reiten formula

$$
\operatorname{Ext}_{\mathbb{C} Q}^{1}(X, Y) \cong D \operatorname{Hom}_{\mathbb{C} Q}(Y, \tau X)
$$

for $X, Y \in \bmod \mathbb{C} Q$. We can identify $\mathbb{C} Q$-modules with their images in $\mathcal{C}(Q)$ by considering the embedding of $\bmod \mathbb{C} Q$ into $\mathcal{C}(Q)$. Each object $M$ in $\mathcal{C}(Q)$ can be 
uniquely decomposed into the form $M=M_{0} \oplus P_{M}[1]=M_{0} \oplus \tau P_{M}$, where $M_{0} \in$ $\bmod \mathbb{C} Q$ and $P_{M}$ is projective in $\bmod \mathbb{C} Q$.

The Caldero-Chapton map of an acyclic quiver $Q$ is the map

$$
X_{?}^{Q}: \operatorname{obj}(\mathcal{C}(Q)) \rightarrow \mathbb{Q}\left(x_{1}, \cdots, x_{n}\right)
$$

defined in 2 by the following rules:

(1) if $M$ is an indecomposable $\mathbb{C} Q$-module, then

$$
X_{M}^{Q}=\sum_{\mathbf{e}} \chi\left(\operatorname{Gr}_{\mathbf{e}}(M)\right) \prod_{i \in Q_{0}} x_{i}^{-\left\langle\mathbf{e}, s_{i}\right\rangle-\left\langle s_{i}, \operatorname{dim} M-\mathbf{e}\right\rangle} ;
$$

(2) if $M=P_{i}[1]$ is the shift of the projective module associated to $i \in Q_{0}$, then

$$
X_{M}^{Q}=x_{i}
$$

(3) for any two objects $M, N$ of $\mathcal{C}_{Q}$, we have

$$
X_{M \oplus N}^{Q}=X_{M}^{Q} X_{N}^{Q}
$$

Without risk of confusion, we can write $X_{\text {? }}$ instead of $X_{\text {? }}^{Q}$. Let $R=\left(r_{i j}\right)$ be a matrix of size $\left|Q_{0}\right| \times\left|Q_{0}\right|$ satisfying

$$
r_{i j}=\operatorname{dim}_{\mathbb{C}} \operatorname{Ext}^{1}\left(S_{i}, S_{j}\right)
$$

for any $i, j \in Q_{0}$. We need the following lemma [14, Lemma 1] to rewrite the Caldero-Chapoton map.

Lemma 1.5. For any $\mathbb{C} Q$-module $M$ without projective summands, we have

$$
(\operatorname{dim} M) R+(\operatorname{dim} \tau M) R^{t r}=\operatorname{dim} M+\operatorname{dim} \tau M .
$$

For a projective $\mathbb{C} Q$-module $P$ and an injective module $I$, we have

$$
(\operatorname{dim} P) R=\operatorname{dim} r a d P, \quad(\operatorname{dim} I) R^{t r}=\operatorname{dim} s o c I .
$$

Following this lemma, we rewrite the above map using the following rules:

$$
X_{\tau P}=X_{P[1]}=x^{\operatorname{dim} P / \operatorname{rad} P}, X_{\tau^{-1} I}=X_{I[-1]}=x^{\operatorname{dim} s o c I}
$$

for any projective $\mathbb{C} Q$-module $P$ and any injective $\mathbb{C} Q$-module $I$;

$$
X_{M}=\sum_{\mathbf{e}} \chi\left(\operatorname{Gr}_{\mathbf{e}}(M)\right) x^{\mathbf{e} R+(\operatorname{dim} M-\mathbf{e}) R^{t r}-\operatorname{dim} M},
$$

where $M$ is a $\mathbb{C} Q$-module and $R^{t r}$ is the transpose of the matrix $R$;

$$
X_{M \oplus P[1]}=X_{M} X_{P[1]}
$$

for any $\mathbb{C} Q$-module $M$ and projective $\mathbb{C} Q$-module $P$.

Here, for $v=\left(v_{1}, \cdots, v_{n}\right) \in \mathbb{Z}^{n}$, we set

$$
x^{v}=x_{1}^{v_{1}} \cdots x_{n}^{v_{n}} .
$$

The following proposition shows that the new rules allow one to deduce the third rule in the definition of the Caldero-Chapoton map. It is actually a degeneration form of the Green formula (see [8, Theorem 5.7]).

Proposition 1.6 ([2, Proposition 3.6]). For any $M, N \in \bmod \mathbb{C} Q$, we have $X_{M \oplus N}$ $=X_{M} X_{N}$. 
For any $M \in \bmod \mathbb{C} Q$, we say that $P_{0}$ is the maximal projective direct summand of $M$ if $M \simeq M^{\prime} \oplus P_{0}$ as the $\mathbb{C} Q$-modules and $M^{\prime}$ does not contain projective direct summands.

Let $\tilde{A}(Q)$ be the subalgebra of $\mathbb{Q}\left(x_{1}, \cdots, x_{n}\right)$ generated by

$$
\left\{X_{M}, X_{\tau P} \mid M, P \in \operatorname{ind}(\bmod \mathbb{C} Q) \text { and } P \text { is projective }\right\}
$$

and $A(Q)$ the subalgebra of $\tilde{A}(Q)$ generated by

$$
\left\{X_{M}, X_{\tau P} \mid M, P \in \operatorname{ind}(\bmod \mathbb{C} Q), P \text { is projective and } \operatorname{Ext}^{1}(M, M)=0\right\} .
$$

The algebra $A(Q)$ is called the cluster algebra associated to $Q$. If $Q$ is of finite type, then $\tilde{A}(Q)$ is just the cluster algebra $A(Q)$ as shown in [4. We note that the relation between $\tilde{A}(Q)$ and $A(Q)$ is generally different from the relation between the Ringel-Hall algebra and the composition algebra for $Q$. For example, $\tilde{A}(Q)$ is equal to $A(Q)$ when $Q$ is a Kronecker quiver (see Section 4.2).

\section{MORPHISMS OF VARIETIES INDUCED BY KERNELS, COKERNELS And THE AUslander-Reiten translation}

The cluster multiplication theorem for arbitrary type in Section 4 will be expressed in the context of $\mathbb{C} Q$-modules. In the sequel, we will only consider the restriction of the Auslander-Reiten translation $\tau$ to $\bmod \mathbb{C} Q$, rather than the cluster category. We use the same notation without risk of confusion. Hence, $\tau P=$ $\tau^{-1} I=0$ for any projective module $P$ and injective module $I$. In this section, we define constructible morphisms lifting the constructions of kernels, cokernels and the Auslander-Reiten translation. These morphisms guarantee that we can use Proposition 1.1.

2.1. Morphisms lifting kernels and cokernels. Let $\left(\mathbb{C}^{\mathbf{d}}, x\right)$ and $\left(\mathbb{C}^{\mathbf{d}^{\prime}}, x^{\prime}\right)$ be two $\mathbb{C} Q$-modules. In this subsection, for any $f \in \operatorname{Hom}_{\mathbb{C} Q}\left(\left(\mathbb{C}^{\mathbf{d}}, x\right),\left(\mathbb{C}^{\mathbf{d}^{\prime}}, x^{\prime}\right)\right)$, we will describe $\operatorname{ker} f, \operatorname{Im} f$ and coker $f$ under geometric context. The main obstacle is that the underlying spaces for $\operatorname{ker} f, \operatorname{Im} f$ and $\operatorname{coker} f$ are not of the form $\mathbb{C}^{\mathbf{e}}$ for some dimension vector e. First, we deal with the case of vector spaces.

Let $\mathbb{C}^{d}$ and $\mathbb{C}^{d^{\prime}}$ be two vector spaces of dimension $d$ and $d^{\prime}$, respectively. Let $M_{d^{\prime} \times d}$ be the set of all matrices of size $d^{\prime} \times d$. Then $M_{d^{\prime} \times d}=\operatorname{Hom}\left(\mathbb{C}^{d}, \mathbb{C}^{d^{\prime}}\right)$ and $M_{d^{\prime} \times d}=\bigsqcup_{r} M_{d^{\prime} \times d}(r)$, where $M_{d^{\prime} \times d}(r)$ consists of all matrices of rank $r$. For any $A=\left(a_{i j}\right) \in M_{d^{\prime} \times d}(r)$, let us denote the $r \times r$ submatrix of $A$ formed by the rows $1 \leq i_{1}<\cdots<i_{r} \leq d^{\prime}$ and the columns $1 \leq j_{1}<\cdots<j_{r} \leq d$ by $\triangle_{\left(i_{1}, \cdots, i_{r} ; j_{1}, \cdots, j_{r}\right)}(A)$. For every pair of multi-indices $I=\left\{i_{1}, \cdots, i_{r}\right\} \subseteq\left\{1, \cdots, d^{\prime}\right\}$ and $J=\left\{j_{1}, \cdots, j_{r}\right\} \subseteq\{1, \cdots, d\}$, we define $M_{d^{\prime} \times d}(r, I, J)$ to be the subset of $M_{d^{\prime} \times d}(r)$ consisting of the matrices $A$ which satisfy $A \notin M_{d^{\prime} \times d}\left(I^{\prime}, J^{\prime}\right)$ for any $I^{\prime}<I$ or $I^{\prime}=I, J^{\prime}<J$ and $\operatorname{det} \triangle_{\left(i_{1}, \cdots, i_{r} ; j_{1}, \cdots, j_{r}\right)}(A) \neq 0$. Here $I^{\prime}<I$ is the common lexicographic order. We have a finite stratification of $M_{d^{\prime} \times d}(r)$, i.e.,

$$
M_{d^{\prime} \times d}(r)=\bigsqcup_{(I, J)} M_{d^{\prime} \times d}(r, I, J) .
$$

In particular, if $d<d^{\prime}$ and $r=d$, this gives a finite stratification of the Grassmannian $\mathrm{Gr}_{d}\left(\mathbb{C}^{d^{\prime}}\right)$ consisting of all $d$-dimensional subspaces of $\mathbb{C}^{d^{\prime}}$. Indeed, for any $I=\left\{i_{1}, \cdots, i_{d}\right\} \subset\left\{1, \cdots, d^{\prime}\right\}$ and $J=\{1, \cdots, d\}$, let $M_{d^{\prime} \times d}^{g}(I)$ be the subset of 
$M_{d^{\prime} \times d}(d, I, J)$ consisting of the matrices $A$ satisfying the fact that $\triangle_{(I ; J)}(A)$ are identity matrices. Then there is a finite stratification

$$
\operatorname{Gr}_{d}\left(\mathbb{C}^{d^{\prime}}\right)=\bigsqcup_{I} M_{d^{\prime} \times d}^{g}(I) .
$$

For any $A \in M_{d^{\prime} \times d}(d, I, J)$, we substitute the identity matrix for the submatrix $\triangle_{(I ; J)}(A)$ and then $A$ corresponds to a unqiue matrix $A^{\prime} \in M_{d^{\prime} \times d}^{g}(I)$.

For every pair of multi-indices $I=\left\{i_{1}, \cdots, i_{r}\right\}$ and $J=\left\{j_{1}, \cdots, j_{r}\right\}$, we will define the following morphism of varieties:

$$
\begin{gathered}
\Upsilon_{(r, I, J)}^{1}: M_{d^{\prime} \times d}(r, I, J) \rightarrow M_{d \times(d-r)}(d-r), \\
\Upsilon_{(r, I, J)}^{2}: M_{d^{\prime} \times d}(r, I, J) \rightarrow \operatorname{Gr}_{d-r}\left(\mathbb{C}^{d}\right), \\
\Omega_{(r, I, J)}^{1}: M_{d^{\prime} \times d}(r, I, J) \rightarrow M_{\left(d^{\prime}-r\right) \times d^{\prime}}\left(d^{\prime}-r\right),
\end{gathered}
$$

and

$$
\Omega_{(r, I, J)}^{2}: M_{d^{\prime} \times d}(r, I, J) \rightarrow \mathrm{Gr}_{r}\left(\mathbb{C}^{d^{\prime}}\right)
$$

Let $P_{i j}(k)$ be the elementary matrix of size $k \times k$ transposing the $i$-th row and the $j$-th row. Set $P_{I}\left(d^{\prime}\right)=P_{r, i_{r}}\left(d^{\prime}\right) \cdots P_{1, i_{1}}\left(d^{\prime}\right)$ and $P_{J}(d)=P_{r, j_{r}}(d) \cdots P_{1, j_{1}}(d)$. Then we have

$$
P_{I}\left(d^{\prime}\right) A P_{J}(d) \in M_{d^{\prime} \times d}(r,(1, \cdots, r)(1, \cdots, r))
$$

for any matrix $A \in M_{d^{\prime} \times d}(r, I, J)$. Consequently, the matrix $P_{I}\left(d^{\prime}\right) A P_{J}(d)$ has the form $\left(\begin{array}{cc}A_{1} & A_{2} \\ A_{3} & A_{4}\end{array}\right)$ with an invertible $r \times r$ matrix $A_{1}$ and $A_{4}=A_{3} A_{1}^{-1} A_{2}=$ $A_{2} A_{1}^{-1} A_{3}$, the matrix $P_{J}(d)\left(\begin{array}{c}-A_{1}^{-1} A_{2} \\ I_{d-r}\end{array}\right)$ determines the solution space $\left\{x \in \mathbb{C}^{d} \mid\right.$ $A x=0\}$, and the matrix $\left(-A_{3} A_{1}^{-1}, I_{d^{\prime}-r}\right) P_{I}\left(d^{\prime}\right)$ determines the solution space $\left\{x \in \mathbb{C}^{d} \mid x A=0\right\}$. We define

$$
\begin{aligned}
& \Upsilon_{(r, I, J)}^{1}(A)=P_{J}(d)\left(\begin{array}{c}
-A_{1}^{-1} A_{2} \\
I_{d-r}
\end{array}\right), \\
& \Omega_{(r, I, J)}^{1}(A)=\left(-A_{3} A_{1}^{-1}, I_{d^{\prime}-r}\right) P_{I}\left(d^{\prime}\right) .
\end{aligned}
$$

Assume that $P_{J}(d)\left(\begin{array}{c}-A_{1}^{-1} A_{2} \\ I_{d-r}\end{array}\right) \in M_{d \times(d-r)}\left(d-r, I^{\prime}, J^{\prime}\right)$ for some $I^{\prime} \subset\{1, \cdots, d\}$ and $J^{\prime}=(1, \cdots, d-r)$. Then we define $\Upsilon_{(r, I, J)}^{2}(A)$ to be the unique matrix in $M_{d \times(d-r)}^{g}\left(I^{\prime}\right)$ with which the matrix $P_{J}(d)\left(\begin{array}{c}-A_{1}^{-1} A_{2} \\ I_{d-r}\end{array}\right)$ corresponds. Similarly, we define $\Omega_{(r, I, J)}^{2}(A)$ to be the unique matrix in $M_{d^{\prime} \times r}^{g}(I)$ with which the submatrix $\triangle_{\left(1, \cdots, d^{\prime} ; j_{1}, \cdots, j_{r}\right)}(A)$ of $A$ corresponds. Hence, for any $A \in M_{d^{\prime} \times d}(r, I, J)$, we have a long exact sequence of $\mathbb{C}$-spaces

$$
0 \longrightarrow \mathbb{C}^{d-r} \stackrel{\Upsilon_{(r, I, J)}^{1}(A)}{\longrightarrow} \mathbb{C}^{d} \stackrel{A}{\longrightarrow} \mathbb{C}^{d^{\prime}} \stackrel{\Omega_{(r, I, J)}^{1}(A)}{\longrightarrow} \mathbb{C}^{d^{\prime}-r} \longrightarrow 0 .
$$

Now, we consider $\mathbb{C} Q$-modules. Let $\left(\mathbb{C}^{\mathbf{d}}, x\right)$ and $\left(\mathbb{C}^{\mathbf{d}^{\prime}}, x^{\prime}\right)$ be two $\mathbb{C} Q$-modules with dimension vectors $\mathbf{d}$ and $\mathbf{d}^{\prime}$, respectively. For any morphism of $\mathbb{C} Q$-modules $f:\left(\mathbb{C}^{\mathbf{d}}, x\right) \rightarrow\left(\mathbb{C}^{\mathbf{d}^{\prime}}, x^{\prime}\right)$, we have $f=\left(f_{i}\right)_{i \in Q_{0}}$ with $f_{i} \in M_{d_{i}^{\prime} \times d_{i}}$ and $f_{t(\alpha)} x_{\alpha}=$ 
$x_{\alpha}^{\prime} f_{s(\alpha)}$ for any $i \in Q_{0}$ and $\alpha \in Q_{1}$. There is a finite stratification of $M_{d_{i}^{\prime} \times d_{i}}$ for any $i \in Q_{0}$ as follows:

$$
M_{d_{i}^{\prime} \times d_{i}}=\bigsqcup_{r_{i}, I_{i}, J_{i}} M_{d_{i}^{\prime} \times d_{i}}\left(r_{i}, I_{i}, J_{i}\right) .
$$

Then $\operatorname{Hom}_{\mathbb{C Q}}\left(\left(\mathbb{C}^{\mathbf{d}}, x\right),\left(\mathbb{C}^{\mathbf{d}^{\prime}}, x^{\prime}\right)\right)$ is a closed subset of

$$
\prod_{i \in Q_{0}} M_{d_{i}^{\prime} \times d_{i}}=\bigsqcup_{\left(\left(r_{i}, I_{i}, J_{i}\right)\right)_{i \in Q_{0}}} \prod_{i \in Q_{0}} M_{d_{i}^{\prime} \times d_{i}}\left(r_{i}, I_{i}, J_{i}\right) .
$$

This induces a finite stratification of $\operatorname{Hom}_{\mathbb{C Q}}\left(\left(\mathbb{C}^{\mathbf{d}}, x\right),\left(\mathbb{C}^{\mathbf{d}^{\prime}}, x^{\prime}\right)\right)$.

For any $i \in Q_{0}$, we fix a pair of multi-indices $I_{i}=\left(k_{i 1}, \cdots, k_{i r_{i}}\right)$ and $J_{i}=$ $\left(l_{i 1}, \cdots, l_{i r_{i}}\right)$. We have the following morphism of varieties:

$$
\begin{aligned}
& \Upsilon_{\left(\left(r_{i}, I_{i}, J_{i}\right)\right)_{i} \in Q_{0}}^{1}:=\prod_{i \in Q_{0}} \Upsilon_{\left(r_{i}, I_{i}, J_{i}\right)}^{1}: \prod_{i \in Q_{0}} M_{d_{i}^{\prime} \times d_{i}}\left(r_{i}, I_{i}, J_{i}\right) \rightarrow \prod_{i \in Q_{0}} M_{d_{i} \times\left(d_{i}-r_{i}\right)}\left(d_{i}-r_{i}\right), \\
& \Upsilon_{\left(\left(r_{i}, I_{i}, J_{i}\right)\right)_{i \in Q_{0}}}^{2}:=\prod_{i \in Q_{0}} \Upsilon_{\left(r_{i}, I_{i}, J_{i}\right)}^{2}: \prod_{i \in Q_{0}} M_{d_{i}^{\prime} \times d_{i}}\left(r_{i}, I_{i}, J_{i}\right) \rightarrow \prod_{i \in Q_{0}} \operatorname{Gr}_{d_{i}-r_{i}}\left(\mathbb{C}^{d_{i}}\right), \\
& \Omega_{\left(\left(r_{i}, I_{i}, J_{i}\right)\right)_{i \in Q_{0}}}^{1}:=\prod_{i \in Q_{0}} \Omega_{\left(r_{i}, I_{i}, J_{i}\right)}^{1}: \prod_{i \in Q_{0}} M_{d_{i}^{\prime} \times d_{i}}\left(r_{i}, I_{i}, J_{i}\right) \rightarrow \prod_{i \in Q_{0}} M_{\left(d_{i}^{\prime}-r_{i}\right) \times d_{i}^{\prime}}\left(d_{i}^{\prime}-r_{i}\right)
\end{aligned}
$$

and

$$
\Omega_{\left(\left(r_{i}, I_{i}, J_{i}\right)\right)_{i \in Q_{0}}}^{2}:=\prod_{i \in Q_{0}} \Omega_{\left(r_{i}, I_{i}, J_{i}\right)}^{2}: \prod_{i \in Q_{0}} M_{d_{i}^{\prime} \times d_{i}}\left(r_{i}, I_{i}, J_{i}\right) \rightarrow \prod_{i \in Q_{0}} \operatorname{Gr}_{r_{i}}\left(\mathbb{C}^{d_{i}^{\prime}}\right) .
$$

Without loss of generality, we can assume the above $\mathbb{C} Q$-module homomorphism $f \in \prod_{i \in Q_{0}} M_{d_{i}^{\prime} \times d_{i}}\left(r_{i}, I_{i}, J_{i}\right)$. Then we have $\Upsilon_{\left(\left(r_{i}, I_{i}, J_{i}\right)\right)_{i \in Q_{0}}}^{2}(f) \in \operatorname{Gr}_{\mathbf{d}-\mathbf{r}}\left(\left(\mathbb{C}^{\mathbf{d}}, x\right)\right)$ with $\mathbf{r}=\left(r_{i}\right)_{i \in Q_{0}}$. Indeed, since

$$
f_{t(\alpha)} x_{\alpha}\left(\Upsilon_{\left(r_{s(\alpha)}, I_{s(\alpha)}, J_{s(\alpha)}\right)}^{2}\left(f_{s(\alpha)}\right)\right)=x_{\alpha}^{\prime} f_{s(\alpha)}\left(\Upsilon_{\left(r_{s(\alpha)}, I_{s(\alpha)}, J_{s(\alpha)}\right)}^{2}\left(f_{s(\alpha)}\right)\right)=0,
$$

then $x_{\alpha}\left(\Upsilon_{\left(r_{s(\alpha)}, I_{s(\alpha)}, J_{s(\alpha)}\right)}^{2}\left(f_{s(\alpha)}\right)\right) \in \Upsilon_{\left(r_{t(\alpha)}, I_{t(\alpha)}, J_{t(\alpha)}\right)}^{2}\left(f_{t(\alpha)}\right)$. In fact,

$$
\Upsilon_{\left(\left(r_{i}, I_{i}, J_{i}\right)\right)_{i \in Q_{0}}}^{2}(f)=\operatorname{ker} f .
$$

As discussed in the case of a matrix, for any $i \in Q_{0}$, we assume $P_{I_{i}}\left(d_{i}^{\prime}\right) f_{i} P_{J_{i}}\left(d_{i}\right)$ has the form $\left(\begin{array}{ll}A_{1}\left(f_{i}\right) & A_{2}\left(f_{i}\right) \\ A_{3}\left(f_{i}\right) & A_{4}\left(f_{i}\right)\end{array}\right)$ with an invertible $r_{i} \times r_{i}$ matrix $A_{1}\left(f_{i}\right)$ and $A_{4}\left(f_{i}\right)=A_{3}\left(f_{i}\right)\left(A_{1}\left(f_{i}\right)\right)^{-1} A_{2}\left(f_{i}\right)=A_{2}\left(f_{i}\right)\left(A_{1}\left(f_{i}\right)\right)^{-1} A_{3}\left(f_{i}\right)$. Then we have a $\mathbb{C} Q$ module $\left(\mathbb{C}^{\mathbf{d}-\mathbf{r}}, y\right)$ isomorphic to $\operatorname{ker} f$ such that

$$
y_{\alpha}=\left(\begin{array}{ll}
0 & I_{d_{t(\alpha)}-r_{t(\alpha)}}
\end{array}\right) P_{J_{t(\alpha)}}\left(d_{t(\alpha)}\right) x_{\alpha} P_{J_{s(\alpha)}}\left(d_{s(\alpha)}\right)\left(\begin{array}{c}
-\left(A_{1}\left(f_{s(\alpha)}\right)\right)^{-1} A_{2}\left(f_{s(\alpha)}\right) \\
I_{d_{s(\alpha)}-r_{s(\alpha)}}
\end{array}\right) .
$$

Now we can write the following left exact sequence of $\mathbb{C} Q$-modules:

$$
0 \longrightarrow\left(\mathbb{C}^{\mathbf{d}-\mathbf{r}}, y\right) \stackrel{\Upsilon_{\left(\left(r_{i}, I_{i}, J_{i}\right)\right)_{i \in Q_{0}}}^{1}(f)}{\longrightarrow}\left(\mathbb{C}^{\mathbf{d}}, x\right) \stackrel{f}{\longrightarrow}\left(\mathbb{C}^{\mathbf{d}^{\prime}}, x^{\prime}\right) .
$$

By similar discussion, we obtain

$$
\Omega_{\left(\left(r_{i}, I_{i}, J_{i}\right)\right)_{i \in Q_{0}}}^{2}(f)=\operatorname{Im} f
$$


and a $\mathbb{C} Q$-module $\left(\mathbb{C}^{\mathbf{d}^{\prime}-\mathbf{r}}, y^{\prime}\right)$ isomorphic to coker $f$ satisfying the following right exact sequence:

$$
\left(\mathbb{C}^{\mathbf{d}}, x\right) \stackrel{f}{\longrightarrow}\left(\mathbb{C}^{\mathbf{d}^{\prime}}, x^{\prime}\right) \stackrel{\Omega_{\left(\left(r_{i}, I_{i}, J_{i}\right)\right)_{i \in Q_{0}}(f)}^{\longrightarrow}}{\longrightarrow}\left(\mathbb{C}^{\mathbf{d}^{\prime}-\mathbf{r}}, y^{\prime}\right) \longrightarrow 0 .
$$

Therefore, we have the following proposition.

Proposition 2.1. For any $\mathbb{C} Q$-modules $\left(\mathbb{C}^{\mathbf{d}}, x\right)$ and $\left(\mathbb{C}^{\mathbf{d}^{\prime}}, x^{\prime}\right)$, there are the following maps whose restrictions to the strata are morphisms of varieties:

$$
\begin{aligned}
& \Upsilon^{1}: \operatorname{Hom}_{\mathbb{C Q}}\left(\left(\mathbb{C}^{\mathbf{d}}, x\right),\left(\mathbb{C}^{\mathbf{d}^{\prime}}, x^{\prime}\right)\right) \rightarrow \prod_{\mathbf{e}} \operatorname{Inj}\left(\mathbb{E}_{\mathbf{e}}(Q),\left(\mathbb{C}^{\mathbf{d}}, x\right)\right), \\
& \Upsilon^{2}: \operatorname{Hom}_{\mathbb{C} Q}\left(\left(\mathbb{C}^{\mathbf{d}}, x\right),\left(\mathbb{C}^{\mathbf{d}^{\prime}}, x^{\prime}\right)\right) \rightarrow \prod_{\mathbf{e}} \operatorname{Gr}_{\mathbf{e}}\left(\left(\mathbb{C}^{\mathbf{d}}, x\right)\right), \\
& \Omega^{1}: \operatorname{Hom}_{\mathbb{C Q}}\left(\left(\mathbb{C}^{\mathbf{d}}, x\right),\left(\mathbb{C}^{\mathbf{d}^{\prime}}, x^{\prime}\right)\right) \rightarrow \prod_{\mathbf{f}} \operatorname{Surj}\left(\left(\mathbb{C}^{\mathbf{d}^{\prime}}, x^{\prime}\right), \mathbb{E}_{\mathbf{f}}(Q)\right), \\
& \Omega^{2}: \operatorname{Hom}_{\mathbb{C} Q}\left(\left(\mathbb{C}^{\mathbf{d}}, x\right),\left(\mathbb{C}^{\mathbf{d}^{\prime}}, x^{\prime}\right)\right) \rightarrow \prod_{\mathbf{e}^{\prime}} \operatorname{Gr}_{\mathbf{e}^{\prime}}\left(\left(\mathbb{C}^{\mathbf{d}^{\prime}}, x^{\prime}\right)\right),
\end{aligned}
$$

where $\operatorname{Inj}\left(\mathbb{E}_{\mathbf{e}}(Q),\left(\mathbb{C}^{\mathbf{d}}, x\right)\right)$ is the set

$$
\left\{\left(\left(\mathbb{C}^{\mathbf{e}}, y\right), g\right) \mid g:\left(\mathbb{C}^{\mathbf{e}}, y\right) \rightarrow\left(\mathbb{C}^{\mathbf{d}}, x\right) \text { is an injective } \mathbb{C} Q \text {-homomorphism }\right\}
$$

and $\operatorname{Surj}\left(\left(\mathbb{C}^{\mathbf{d}^{\prime}}, x^{\prime}\right), \mathbb{E}_{\mathbf{f}}(Q)\right)$ is the set

$$
\left\{\left(\left(\mathbb{C}^{\mathbf{f}}, y^{\prime}\right), g^{\prime}\right) \mid g^{\prime}:\left(\mathbb{C}^{\mathbf{d}^{\prime}}, x^{\prime}\right) \rightarrow\left(\mathbb{C}^{\mathbf{f}}, y^{\prime}\right) \text { is a surjective } \mathbb{C} Q \text {-homomorphism }\right\}
$$

satisfying the fact that for any $f \in \operatorname{Hom}_{\mathbb{C} Q}\left(\left(\mathbb{C}^{\mathbf{d}}, x\right),\left(\mathbb{C}^{\mathbf{d}^{\prime}}, x^{\prime}\right)\right)$, there exists a long exact sequence of $\mathbb{C} Q$-modules

$$
0 \longrightarrow\left(\mathbb{C}^{\mathbf{e}}, y\right) \stackrel{\Upsilon^{1}(f)}{\longrightarrow}\left(\mathbb{C}^{\mathbf{d}}, x\right) \stackrel{f}{\longrightarrow}\left(\mathbb{C}^{\mathbf{d}^{\prime}}, x^{\prime}\right) \stackrel{\Omega^{1}(f)}{\longrightarrow}\left(\mathbb{C}^{\mathbf{f}}, y^{\prime}\right) \longrightarrow 0 .
$$

By the proof of Proposition 2.1, we also have the following corollary.

Corollary 2.2. For any $\mathbb{C} Q$-modules $\left(\mathbb{C}^{\mathbf{d}}, x\right)$ and dimension vector $\mathbf{e}$, there are the following maps whose restrictions to the strata are morphisms of varieties:

$$
\Upsilon_{0}: \operatorname{Gr}_{\mathbf{e}}\left(\left(\mathbb{C}^{\mathbf{d}}, x\right)\right) \rightarrow \mathbb{E}_{\mathbf{e}}(Q) \quad \text { and } \quad \Omega_{0}: \operatorname{Gr}_{\mathbf{e}}\left(\left(\mathbb{C}^{\mathbf{d}}, x\right)\right) \rightarrow \mathbb{E}_{\mathbf{d}-\mathbf{e}}(Q)
$$

such that for any $M \in \mathrm{Gr}_{\mathbf{e}}\left(\left(\mathbb{C}^{\mathbf{d}}, x\right)\right)$, as the $\mathbb{C} Q$-modules, $\Upsilon_{0}(M) \cong M$ and $\Omega_{0}(M)$ $\cong\left(\mathbb{C}^{\mathbf{d}}, x\right) / M$.

2.2. Morphisms lifting the Auslander-Reiten translation. In this subsection, we will describe the Auslander-Reiten translation $\tau$ under geometric context. Let $\Phi^{+}, \Phi^{-}$be the Coxeter functors introduced by Bernsetein, Gelfand and Ponomarev. We denote by $T$ the endofunctor of $\bmod \mathbb{C} Q$ sending $\left(\mathbb{C}^{\mathbf{d}}, x\right)$ to $\left(\mathbb{C}^{\mathbf{d}},-x\right)$. Then the functor $T \Phi^{+}$on $\bmod \mathbb{C} Q$ is just the Auslander-Reiten translation $\tau$ on $\bmod \mathbb{C} Q$ (see [10, Proposition 5.3]).

Given any $\mathbb{C} Q$-module $\left(\mathbb{C}^{\mathbf{d}}, x\right) \in \mathbb{E}_{\mathbf{d}}$, the representation

$$
\Phi^{+}\left(\mathbb{C}^{\mathbf{d}}, x\right):=\left(\mathbb{C}^{\mathbf{e}}, y\right)
$$

can be constructed inductively as described in [26]. Let us recall it. Since $Q$ is acyclic, one can define a partial order on $Q_{0}$ such that for any arrow $\beta, s(\beta)>t(\beta)$. Assume that the dimension $e_{j}$ with $j<i$, the linear maps $h_{\beta}: \mathbb{C}^{e_{t(\beta)}} \rightarrow \mathbb{C}^{d_{s(\beta)}}$ for 
all $\beta \in Q_{1}$ with $s(\beta) \leq i$ and the maps $y_{\alpha}: \mathbb{C}^{e_{s(\alpha)}} \rightarrow \mathbb{C}^{e_{t(\alpha)}}$ for all $\alpha \in Q_{1}$ with $s(\alpha)<i$ are defined. Then we have the sequence

$$
0 \longrightarrow \mathbb{C}^{e_{i}} \stackrel{\Upsilon^{1}\left(\left(x_{\alpha}, h_{\beta}\right)_{\alpha, \beta}\right)}{\longrightarrow} \bigoplus_{t(\alpha)=i} \mathbb{C}^{d_{s(\alpha)}} \oplus \bigoplus_{s(\beta)=i} \mathbb{C}^{e_{t(\beta)}} \stackrel{\left(x_{\alpha}, h_{\beta}\right)_{\alpha, \beta}}{\longrightarrow} \mathbb{C}^{d_{i}},
$$

where $e_{i}$ is the dimension of the kernel of the map $\left(x_{\alpha}, h_{\beta}\right)_{\alpha, \beta}$ and $\Upsilon^{1}$ is defined as in Section 2.1. Now we define the map $y_{\beta}: \mathbb{C}^{e_{s(\beta)}} \rightarrow \mathbb{C}^{e_{t(\beta)}}$ for any $\beta$ with $s(\beta)=i$ to be the composition

$$
\mathbb{C}^{e_{i}} \stackrel{\Upsilon^{1}\left(\left(x_{\alpha}, h_{\beta}\right)_{\alpha, \beta}\right)}{\longrightarrow} \bigoplus_{t(\alpha)=i} \mathbb{C}^{d_{s(\alpha)}} \oplus \bigoplus_{s(\beta)=i} \mathbb{C}^{e_{t(\beta)}} \longrightarrow \mathbb{C}^{e_{t(\beta)}}
$$

where the second map is the projection. Inductively we obtain the representation $\tau\left(\mathbb{C}^{\mathbf{d}}, x\right)=T \Phi^{+}\left(\mathbb{C}^{\mathbf{d}}, x\right)=\left(\mathbb{C}^{\mathbf{e}},-y\right)$. We write $\tau(\mathbf{d})=\mathbf{e}$. The geometric construction of $\Phi^{-}\left(\right.$also $\left.\tau^{-}\right)$is similar. For any $\mathbb{C} Q$-module $(\mathbb{C} \mathbf{d}, x) \in \mathbb{E}_{\mathbf{d}}$, let $\left(\mathbb{C}^{\mathbf{d}^{+}}, x^{+}\right)$ and $P_{0}(\mathbf{d}, x)$ be its maximal non-projective summand and the maximal projective summand, respectively, i.e., $\left(\mathbb{C}^{\mathbf{d}}, x\right)=\left(\mathbb{C}^{\mathbf{d}^{+}}, x^{+}\right) \oplus P_{0}(\mathbf{d}, x)$ satisfying the fact that $\left(\mathbb{C}^{\mathbf{d}^{+}}, x^{+}\right)$contains no projective summands and $P_{0}$ is projective. In fact, we have the isomorphism of $\mathbb{C} Q$-modules

$$
\tau^{-1} \tau\left(\mathbb{C}^{\mathbf{d}}, x\right) \cong\left(\mathbb{C}^{\mathbf{d}^{+}}, x^{+}\right) .
$$

We can explicitly write the submodule $(V, x):=\tau^{-1} \tau\left(\mathbb{C}^{\mathbf{d}}, x\right)$ of $\left(\mathbb{C}^{\mathbf{d}}, x\right)$. The space $V_{i}$ is just the image of $\left(x_{\alpha}, h_{\beta}\right)_{\alpha, \beta}$ for any $i \in Q_{0}$ in the sequence (2.1). Dually, we denote by $\left(\mathbb{C}^{\mathbf{d}^{-}}, x^{-}\right)$and $I_{0}(\mathbf{d}, x)$ the maximal non-injective summand and the maximal injective summand of $\left(\mathbb{C}^{\mathbf{d}}, x\right)$, respectively. Then

$$
\tau \tau^{-1}\left(\mathbb{C}^{\mathbf{d}}, x\right) \cong\left(\mathbb{C}^{\mathbf{d}^{-}}, x^{-}\right) .
$$

The above construction and its duality yield the following two propositions.

Proposition 2.3. For any dimension vector $\mathbf{d}$, there exists a map

$$
\phi^{+}: \mathbb{E}_{\mathbf{d}} \rightarrow \prod_{\tau\left(\mathbf{d}^{+}\right)} \mathbb{E}_{\tau\left(\mathbf{d}^{+}\right)}
$$

such that restrictions of $\phi^{+}$to the strata are morphisms of varieties and $\phi^{+}((\mathbb{C} \mathbf{d}, x))$ $=\tau\left(\mathbb{C}^{\mathbf{d}}, x\right)$.

Dually, we have

Proposition 2.4. For any dimension vector $\mathbf{d}$, there exists a map

$$
\phi^{-}: \mathbb{E}_{\mathbf{d}} \rightarrow \prod_{\tau^{-1}\left(\mathbf{d}^{-}\right)} \mathbb{E}_{\tau^{-1}\left(\mathbf{d}^{-}\right)}
$$

such that restrictions of $\phi^{-}$to the strata are morphisms of varieties and $\phi^{-}\left(\left(\mathbb{C}^{\mathbf{d}}, x\right)\right)$ $=\tau^{-1}\left(\mathbb{C}^{\mathbf{d}}, x\right)$.

Let $f=\left(f_{i}\right)_{i \in Q_{0}}:\left(\mathbb{C}^{\mathbf{d}}, x\right) \rightarrow\left(\mathbb{C}^{\mathbf{d}^{\prime}}, x^{\prime}\right)$ be any morphism of $\mathbb{C} Q$-modules. Let $\Phi^{+}\left(\left(\mathbb{C}^{\mathbf{d}}, x\right)\right)=\left(\mathbb{C}^{\mathbf{e}}, y\right)$ and $\Phi^{+}\left(\left(\mathbb{C}^{\mathbf{d}^{\prime}}, x^{\prime}\right)\right)=\left(\mathbb{C}^{\mathbf{e}^{\prime}}, y^{\prime}\right)$. Then we can inductively construct the maps $g=\left(g_{i}\right)_{i \in Q_{0}}:\left(\mathbb{C}^{\mathbf{e}}, y\right) \rightarrow\left(\mathbb{C}^{\mathbf{e}^{\prime}}, y^{\prime}\right)$ by the following commutative 
diagram:

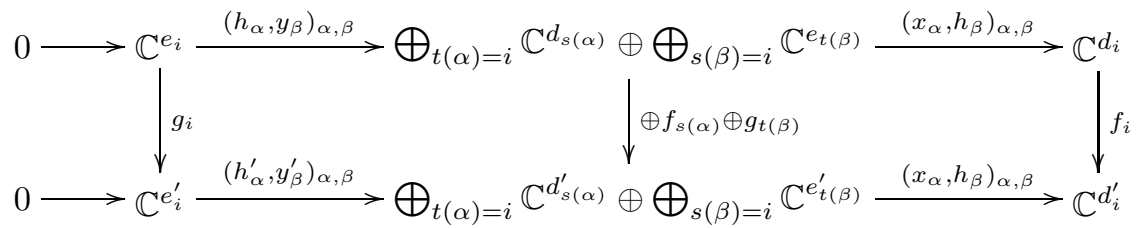

The commutativity of the diagram guarantees that $g=\left(g_{i}\right)$ is the morphism of $\mathbb{C} Q$-modules. Hence, by using Proposition 2.1, Corollary 2.2, and Propositions 2.3 and 2.4, we have the following result.

Proposition 2.5. For any dimension vectors $\mathbf{d}$, there exists a map

$$
\operatorname{Gr}_{\mathbf{e}}\left(\left(\mathbb{C}^{\mathbf{d}}, x\right)\right) \stackrel{g_{\tau}}{\longrightarrow} \operatorname{Gr}_{\tau(\mathbf{e})}\left(\left(\mathbb{C}^{\tau(\mathbf{d})}, \tau x\right)\right)
$$

whose restrictions of the strata are morphisms of varieties.

\section{THE HIGHER ORDER ASSOCIATIVITY}

In [29, Toën associated an associative algebra (called the derived Hall algebra) to a dg category over a finite field $k$. In particular, we have the derived Hall algebra $\mathcal{D H}(Q)$ for the derived category $\mathcal{D}^{b}(\bmod k Q)$. Let $u_{X}$ denote the isomorphism class of $X \in \mathcal{D}^{b}(\bmod k Q)$. The algebra $\mathcal{D H}(Q)$ is an associative algebra whose underlying $\mathbb{Q}$-vector space has a basis given by the $u_{X}$, where $X$ runs through the isomorphism classes of objects of the bounded derived category of finite-dimensional $k Q$-modules. The associative multiplication contains a non-trivial case as follows. For any $L_{1}, L_{2} \in \bmod k Q$, we have

$$
u_{L_{2}} * u_{L_{1}[1]}=\sum_{[\theta], \theta: L_{1} \rightarrow L_{2}} g_{L_{1}[1] L_{2}}^{K[1] \oplus C} u_{K[1] \oplus C}
$$

where $g_{L_{1}[1] L_{2}}^{K[1] \oplus} \in \mathbb{Q}$ is called the derived Hall number and $K=\operatorname{ker} \theta, C=\operatorname{coker} \theta$ and $[\theta]$ is the equivalence class of $\theta$. Here $\theta_{1}$ is equivalent to $\theta_{2}$ if there exist automorphisms $a_{L_{1}}$ and $a_{L_{2}}$ such that $\theta_{1} a_{L_{1}}=a_{L_{2}} \theta_{2}$. The above equation involves the following exact sequence:

$$
0 \longrightarrow K \longrightarrow L_{1} \stackrel{\theta}{\longrightarrow} L_{2} \longrightarrow C \longrightarrow 0 \text {. }
$$

By the associativity of the multiplication of $\mathcal{D H}(Q)$, we have

$$
\left(u_{K_{1}[1]} * u_{L_{2}}\right) * u_{L_{1}[1]}=u_{K_{1}[1]} *\left(u_{L_{2}} * u_{L_{1}[1]}\right)
$$

for any $k Q$-modules $K_{1}, L_{1}, L_{2}$ and

$$
\left(u_{L_{2}} * u_{L_{1}[1]}\right) * u_{C_{2}}=u_{L_{2}} *\left(u_{L_{1}[1]} * u_{C_{2}}\right)
$$


for any $k Q$-modules $C_{2}, L_{1}, L_{2}$. These two equations can be illustrated by the following commutative diagrams:

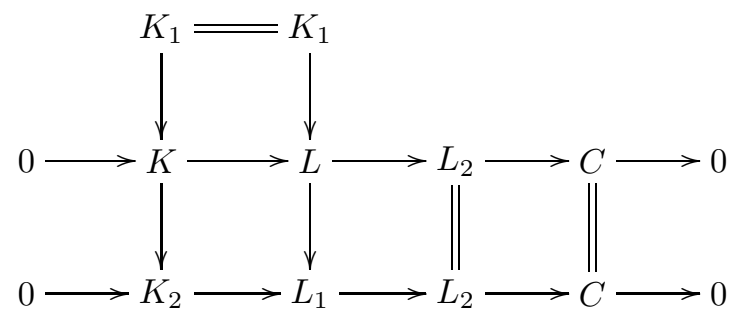

and

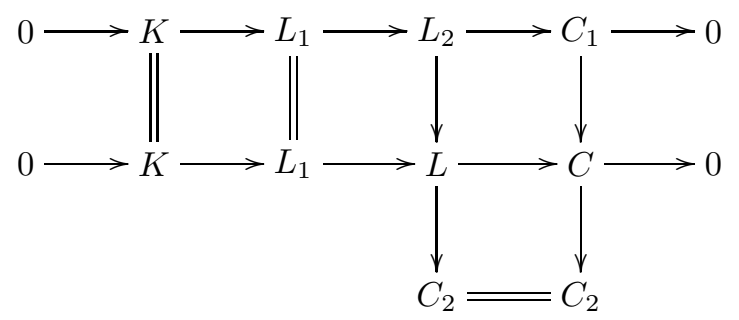

We note that the long exact sequences in the above diagrams can be decomposed into short exact sequences so that the above two equations can be induced by using the associativity of the multiplication of the Ringel-Hall algebra twice. In this section, we will prove an identity analogous to the above associativity of the multiplication of the derived Hall algebra in the context of Euler characteristics. The identity is called the higher order associativity.

3.1. The description of $\operatorname{Ext}_{\mathbb{C} Q}^{1}(M, N)$. Let $M \in \mathbb{E}_{\mathbf{d}_{1}}, N \in \mathbb{E}_{\mathbf{d}_{2}}$ and $m(M, N)$ be the vector space over $\mathbb{C}$ of all tuples $m=(m(\alpha))_{\alpha \in Q_{1}}$ such that linear maps $m(\alpha)$ belong to $\operatorname{Hom}_{\mathbb{C}}\left(M_{s(\alpha)}, N_{t(\alpha)}\right)$ for all $\alpha \in Q_{1}$. We define a linear map $\pi$ : $m(M, N) \rightarrow \operatorname{Ext}^{1}(M, N)$ by mapping $m \in m(M, N)$ to a short exact sequence

$$
\varepsilon: 0 \longrightarrow N \stackrel{\left(\begin{array}{l}
1 \\
0
\end{array}\right)}{\longrightarrow} L(m) \stackrel{\left(\begin{array}{ll}
0 & 1
\end{array}\right)}{\longrightarrow} M \longrightarrow 0,
$$

where, as a vector space, $L(m)$ is the direct sum of $N$ and $M$. For any $\alpha \in Q_{1}$, we put

$$
L(m)_{\alpha}=\left(\begin{array}{cc}
N_{\alpha} & m(\alpha) \\
0 & M_{\alpha}
\end{array}\right) .
$$

We fix a vector space decomposition $m(M, N)=\operatorname{ker} \pi \oplus E(M, N)$. Thus we can identify $\operatorname{Ext}^{1}(M, N)$ with $E(M, N)$. There is a natural $\mathbb{C}^{*}$-action on $E(M, N)$ by defining $t . m=(\operatorname{tm}(\alpha))$ for any $t \in \mathbb{C}^{*}$. This action induces the action of $\mathbb{C}^{*}$ on $\operatorname{Ext}^{1}(M, N)$. Considering the isomorphism of $\mathbb{C} Q$-modules between $L(m)$ and $L(t . m)$, we know that $t . \varepsilon$ is the following short exact sequence:

$$
0 \longrightarrow N \stackrel{\left(\begin{array}{c}
t^{-1} \\
0
\end{array}\right)}{\longrightarrow} L(m) \stackrel{\left(\begin{array}{ll}
0 & 1
\end{array}\right)}{\longrightarrow} M \longrightarrow 0
$$


for any $t \in \mathbb{C}^{*}$. Let $\operatorname{Ext}^{1}(M, N)_{L}$ be the subset of $\operatorname{Ext}^{1}(M, N)$ with the middle term isomorphic to $L$. Then $\operatorname{Ext}^{1}(M, N)_{L}$ can be viewed as a constructible subset of $\operatorname{Ext}^{1}(M, N)$ by identifying $\operatorname{Ext}^{1}(M, N)$ and $E(M, N)$. Define

$$
\operatorname{Ext}^{1}(M, N)_{\mathcal{O}}=\left\{[0 \rightarrow N \rightarrow L \rightarrow M \rightarrow 0] \in \operatorname{Ext}^{1}(M, N) \backslash\{0\} \mid L \in \mathcal{O}\right\},
$$

where the set $\mathcal{O}$ is a $G_{\mathbf{d}_{1}+\mathbf{d}_{2}}$-invariant constructible subset of $\mathbb{E}_{\mathbf{d}_{1}+\mathbf{d}_{2}}$ (see [32] or [12]). It can be identified with

$$
E(M, N)_{\mathcal{O}}=\{m \in E(M, N) \mid L(m) \in \mathcal{O}\},
$$

which is constructible and $\mathbb{C}^{*}$-invariant; see 12 . Hence, $\operatorname{Ext}^{1}(M, N)_{\mathcal{O}}$ can be viewed as a $\mathbb{C}^{*}$-invariant constructible subset of $\operatorname{Ext}^{1}(M, N) \backslash\{0\}$.

3.2. The higher order associativity. Let $M, N \in \bmod \mathbb{C} Q$ and $\tau$ be the Auslander-Reiten translation on $\bmod \mathbb{C} Q$. We assume that $M$ contains no projective summands. Note that for any $X \in \bmod \mathbb{C} Q$, there is a decomposition of $\mathbb{C} Q$-modules

$$
X \cong \tau\left(\tau^{-1} X\right) \oplus X / \tau\left(\tau^{-1} X\right)
$$

with $X / \tau\left(\tau^{-1} X\right)$ isomorphic to an injective $\mathbb{C} Q$-module. For dimension vectors $\mathbf{d}_{1}, \mathbf{d}_{2}$ and $\mathbf{e}_{1}, \mathbf{e}_{2}$, we consider the sets

$$
\begin{aligned}
\operatorname{DEF}_{\mathbf{e}_{1}, \mathbf{e}_{2}}^{\mathbf{d}_{1}, \mathbf{d}_{2}}(N, \tau M)=\left\{\left(g, V_{1}, U_{1}\right) \mid g \in \operatorname{Hom}_{\mathbb{C Q}}(N, \tau M), \operatorname{dimker} g=\mathbf{d}_{1},\right. \\
\left.\operatorname{dim} \tau^{-1}(\operatorname{coker} g)=\mathbf{d}_{2}, V_{1} \in \operatorname{Gr}_{\mathbf{e}_{1}}\left(\Upsilon_{0} \Upsilon^{2}(g)\right), U_{1} \in \operatorname{Gr}_{\mathbf{e}_{2}}\left(\phi^{-} \Omega_{0} \Omega^{2}(g)\right)\right\}
\end{aligned}
$$

and

$$
\begin{aligned}
& \operatorname{DFE}_{\mathbf{e}_{1}, \mathbf{e}_{2}}(N, \tau M) \\
& \quad=\left\{\left(N_{1}, M_{1}, g^{\prime}\right) \mid N_{1} \in \operatorname{Gr}_{\mathbf{e}_{1}}(N), M_{1} \in \operatorname{Gr}_{\mathbf{e}_{2}}(M), g^{\prime} \in \operatorname{Hom}_{\mathbb{C Q}}\left(N / N_{1}, \tau M_{1}\right)\right\} .
\end{aligned}
$$

Here, $\Upsilon_{0}, \Upsilon^{2}, \Omega_{0}, \Omega^{2}$ and $\phi^{-}$were defined as in Section 2. By definition, $\Upsilon_{0} \Upsilon^{2}(g) \in$ $\mathbb{E}_{\mathbf{d}_{1}}, \phi^{-} \Omega_{0} \Omega^{2}(g) \in \mathbb{E}_{\mathbf{d}_{2}}$ and $\Upsilon_{0} \Upsilon^{2}(g) \cong \operatorname{ker} g, \phi^{-} \Omega_{0} \Omega^{2}(g) \cong \tau^{-1}$ (cokerg). We set $U=\phi^{-} \Omega_{0} \Omega^{2}(g)$ and $V=\Upsilon_{0} \Upsilon^{2}(g)$. By the discussion in Section 2.1, there are finite stratifications of $\mathrm{DEF}_{\mathbf{e}_{1}, \mathbf{e}_{2}}^{\mathbf{d}_{1}, \mathbf{d}_{2}}(N, \tau M)$ and $\mathrm{DFE}_{\mathbf{e}_{1}, \mathbf{e}_{2}}(N, \tau M)$.

Remark 3.1. Let's explain the notation for these sets. The letter "D" means "derived". The letters "E" and "F" stand for extension and flag, respectively. Let $M$ and $N$ be two indecomposable $\mathbb{C} Q$-modules. Then (for example, see [1] or 14])

$$
\operatorname{Ext}_{\mathcal{C}(Q)}^{1}(N, M) \cong \operatorname{Ext}_{\mathbb{C} Q}^{1}(N, M) \oplus \operatorname{Hom}_{\mathbb{C} Q}(N, \tau M),
$$

and if $\operatorname{Ext}_{\mathbb{C} Q}^{1}(N, M)=0$, then any $g \in \operatorname{Hom}_{\mathbb{C} Q}(N, \tau M)$ induces an extension of $M$ by $N$ in the cluster category $\mathcal{C}(Q)$ as follows:

$$
M \rightarrow \operatorname{ker} g \oplus \operatorname{coker} g[-1] \rightarrow N \stackrel{g}{\rightarrow} \tau M .
$$

Recall that each principal $\mathbb{C}^{*}$-bundle is locally trivial in the Zariski topology. Let $\pi: P \rightarrow Q$ be such a bundle. Then $(\pi, Q)$ is a geometric quotient for the free action of $\mathbb{C}^{*}$ on $P$ (see [28 and [12]). In the following, we will write $\mathbb{P} x$ for the $\mathbb{C}^{*}$-orbit of $x$ if $x$ belongs to a principal $\mathbb{C}^{*}$-bundle.

Let $\operatorname{Hom}(N, \tau M)\left(\mathbf{d}_{1}, \mathbf{d}_{2}\right)$ be the subset of $\operatorname{Hom}(N, \tau M)$ consisting of the morphism $g$ with $\operatorname{dimker} g=\mathbf{d}_{1}, \operatorname{dim} \tau^{-1}(\operatorname{coker} g)=\mathbf{d}_{2}$. By Corollary 1.4, we have finite subsets $S\left(\mathbf{d}_{1}\right)$ and $S\left(\mathbf{d}_{2}\right)$ of $\mathbb{E}_{\mathbf{d}_{1}}$ and $\mathbb{E}_{\mathbf{d}_{2}}$, respectively, such that

$$
\mathbb{E}_{\mathbf{d}_{1}}=\bigsqcup_{V \in S\left(\mathbf{d}_{1}\right)}\langle V\rangle, \quad \mathbb{E}_{\mathbf{d}_{2}}=\bigsqcup_{U \in S\left(\mathbf{d}_{2}\right)}\langle U\rangle
$$


This yields a finite partition

$$
\operatorname{Hom}(N, \tau M)\left(\mathbf{d}_{1}, \mathbf{d}_{2}\right)=\bigsqcup_{V \in S\left(\mathbf{d}_{1}\right), U \in S\left(\mathbf{d}_{2}\right), I} \operatorname{Hom}(N, \tau M)_{\langle V\rangle \oplus\langle U\rangle \oplus I[-1]},
$$

where $\operatorname{Hom}(N, \tau M)_{\langle V\rangle \oplus\langle U\rangle \oplus I[-1]}$ is

$$
\left\{g \in \operatorname{Hom}(N, \tau M)\left(\mathbf{d}_{1}, \mathbf{d}_{2}\right) \mid \Upsilon_{0} \Upsilon^{2}(g) \in\langle V\rangle, \Omega_{0} \Omega^{2}(g)=\tau U^{\prime} \oplus I,\right.
$$

for some $U^{\prime} \in\langle U\rangle, I$ is an injective $\mathbb{C} Q$-module\}.

Note that $\Omega_{0} \Omega^{2}(g) \cong$ coker $g$.

There is a natural $\mathbb{C}^{*}$-action on

$$
\operatorname{Hom}(N, \tau M)\left(\mathbf{d}_{1}, \mathbf{d}_{2}\right)^{*}:=\operatorname{Hom}(N, \tau M)\left(\mathbf{d}_{1}, \mathbf{d}_{2}\right) \backslash\{0\}
$$

with a principal $\mathbb{C}^{*}$-bundle

$$
\operatorname{Hom}(N, \tau M)\left(\mathbf{d}_{1}, \mathbf{d}_{2}\right)^{*} \rightarrow \mathbb{P H o m}(N, \tau M)\left(\mathbf{d}_{1}, \mathbf{d}_{2}\right) .
$$

Thus by considering the trivial action of $\mathbb{C}^{*}$ on $\mathrm{Gr}_{\mathbf{e}_{1}, \mathbf{e}_{2}}\left(\mathbf{d}_{1}, \mathbf{d}_{2}\right):=\operatorname{Gr}_{\mathbf{e}_{1}}\left(\mathbb{E}_{\mathbf{d}_{1}}\right) \times$ $\mathrm{Gr}_{\mathbf{e}_{2}}\left(\mathbb{E}_{\mathbf{d}_{2}}\right)$, we obtain a new principal $\mathbb{C}^{*}$-bundle (similar to [12, Section 5.4]):

$$
\begin{aligned}
\pi: \operatorname{Hom}(N, \tau M)\left(\mathbf{d}_{1}, \mathbf{d}_{2}\right)^{*} \times \mathrm{Gr}_{\mathbf{e}_{1}, \mathbf{e}_{2}}\left(\mathbf{d}_{1}, \mathbf{d}_{2}\right) \\
\quad \rightarrow \operatorname{Hom}(N, \tau M)\left(\mathbf{d}_{1}, \mathbf{d}_{2}\right)^{*} \times{ }^{\mathbb{C}^{*}} \operatorname{Gr}_{\mathbf{e}_{1}, \mathbf{e}_{2}}\left(\mathbf{d}_{1}, \mathbf{d}_{2}\right) .
\end{aligned}
$$

We note that the action of $\mathbb{C}^{*}$ on $\operatorname{Hom}(N, \tau M)\left(\mathbf{d}_{1}, \mathbf{d}_{2}\right)^{*} \times \mathrm{Gr}_{\mathbf{e}_{1}, \mathbf{e}_{2}}\left(\mathbf{d}_{1}, \mathbf{d}_{2}\right)$ is free. The set $\operatorname{DEF}_{\mathbf{e}_{1}, \mathbf{e}_{2}}^{\mathbf{d}_{1}, \mathbf{d}_{2}}(N, \tau M)$ is its $\mathbb{C}^{*}$-stable constructible subset. This implies that

$$
\operatorname{PDEF}_{\mathbf{e}_{1}, \mathbf{e}_{2}}^{\mathbf{d}_{1}, \mathbf{d}_{2}}(N, \tau M):=\pi\left(\mathrm{DEF}_{\mathbf{e}_{1}, \mathbf{e}_{2}}(N, \tau M)\right)
$$

is again a principal $\mathbb{C}^{*}$-bundle and $\left(\pi, \mathbb{P D E F}_{\mathbf{e}_{1}, \mathbf{e}_{2}}(N, \tau M)\right)$ is a geometric quotient for the action of $\mathbb{C}^{*}$ on $\mathrm{DEF}_{\mathbf{e}_{1}, \mathbf{e}_{2}}(N, \tau M)$ (similar to [12, Section 5.4]). We have a natural projection:

$$
p: \mathbb{P D E F}_{\mathbf{e}_{1}, \mathbf{e}_{2}}^{\mathbf{d}_{1}, \mathbf{d}_{2}}(N, \tau M) \rightarrow \mathbb{P H o m}(N, \tau M)\left(\mathbf{d}_{1}, \mathbf{d}_{2}\right) .
$$

There is a finite partition

$$
\mathbb{P H o m}(N, \tau M)\left(\mathbf{d}_{1}, \mathbf{d}_{2}\right)=\underset{V \in S\left(\mathbf{d}_{1}\right), U \in S\left(\mathbf{d}_{2}\right), I}{ } \mathbb{P H o m}(N, \tau M)_{\langle V\rangle \oplus\langle U\rangle \oplus I[-1]},
$$

where $\mathbb{P} \operatorname{Hom}(N, \tau M)_{\langle V\rangle \oplus\langle U\rangle \oplus I[-1]}$ is the set

$$
\left\{\mathbb{P} g \in \mathbb{P} \operatorname{Hom}(N, \tau M)\left(\mathbf{d}_{1}, \mathbf{d}_{2}\right) \mid \Upsilon_{0} \Upsilon^{2}(g) \in\langle V\rangle, \Omega_{0} \Omega^{2}(g)=\tau U^{\prime} \oplus I,\right.
$$

for some $U^{\prime} \in\langle U\rangle, I$ is an injective $\mathbb{C} Q$-module $\}$.

For any $\mathbb{P} g \in \mathbb{P} \operatorname{Hom}(N, \tau M)\left(\mathbf{d}_{1}, \mathbf{d}_{2}\right)_{\langle V\rangle \oplus\langle U\rangle \oplus I[-1]}$, the Euler characteristic of the fibre $p^{-1}\left(\mathbb{P} g \in \mathbb{P H o m}(N, \tau M)\left(\mathbf{d}_{1}, \mathbf{d}_{2}\right)\right)$ is $\chi\left(\mathrm{Gr}_{\mathbf{e}_{1}}(V)\right) \cdot \chi\left(\mathrm{Gr}_{\mathbf{e}_{2}}(U)\right)$. By Proposition 1.1 we obtain the following lemma.

Lemma 3.2. For fixed dimension vector $\mathbf{d}$, we have

$$
\begin{aligned}
& \quad \sum_{\mathbf{e}_{1}+\mathbf{e}_{2}+\operatorname{dim} M-\mathbf{d}_{2}=\mathbf{d}} \chi\left(\mathbb{P D E F}_{\mathbf{e}_{1}, \mathbf{e}_{2}}^{\mathbf{d}_{1}, \mathbf{d}_{2}}(N, \tau M)\right) \\
& =\sum_{\substack{\mathbf{d}_{1}, \mathbf{d}_{2}, \mathbf{e}_{1}, \mathbf{e}_{2}, U, V, I ; \\
\mathbf{e}_{1}+\mathbf{e}_{2}+\operatorname{dim} M-\mathbf{d}_{2}=\mathbf{d}, U \in S\left(\mathbf{d}_{2}\right), V \in S\left(\mathbf{d}_{1}\right)}} \chi\left(\mathbb{P H o m}(N, \tau M)_{\langle V\rangle \oplus\langle U\rangle \oplus I-1]}\right) \chi\left(\operatorname{Gr}_{\mathbf{e}_{1}}(V)\right) \chi\left(\operatorname{Gr}_{\mathbf{e}_{2}}(U)\right) .
\end{aligned}
$$


There is also a free action of $\mathbb{C}^{*}$ on $\operatorname{DFE}_{\mathbf{e}_{1}, \mathbf{e}_{2}}(N, \tau M)$ defined by

$$
\text { t. }\left(N_{1}, M_{1}, g\right)=\left(N_{1}, M_{1}, t . g\right)
$$

for any $t \in \mathbb{C}^{*}$ and $\left(N_{1}, M_{1}, g\right) \in \operatorname{DFE}_{\mathbf{e}_{1}, \mathbf{e}_{2}}(N, \tau M)$. The orbit space is denoted by $\operatorname{PDFE}_{\mathbf{e}_{1}, \mathbf{e}_{2}}(N, \tau M)$.

Consider a natural projection

$$
q: \operatorname{PDFE}_{\mathbf{e}_{1}, \mathbf{e}_{2}}(N, \tau M) \rightarrow \mathrm{Gr}_{\mathbf{e}_{1}}(N) \times \mathrm{Gr}_{\mathbf{e}_{2}}(M) .
$$

Define $\left\langle\left(N_{1}, M_{1}\right)\right\rangle$ to be

$$
\begin{aligned}
\left\{\left(N_{1}^{\prime}, M_{1}^{\prime}\right) \in \operatorname{Gr}_{\mathbf{e}_{1}}(N) \times \operatorname{Gr}_{\mathbf{e}_{2}}(M) \mid \chi\left(\mathbb{P H o m}\left(N / N_{1}^{\prime}, \tau M_{1}^{\prime}\right)\right)\right. \\
\left.=\chi\left(\mathbb{P H o m}\left(N / N_{1}, \tau M_{1}\right)\right)\right\} .
\end{aligned}
$$

We note that the notation is different from the Euler form $\left\langle N_{1}, M_{1}\right\rangle$ of $N_{1}$ and $M_{1}$. Since $q_{*}\left(1_{\mathbb{P D F E}_{\mathbf{e}_{1}, \mathbf{e}_{2}}(N, \tau M)}\right)$ is a constructible function on $\operatorname{Gr}_{\mathbf{e}_{1}}(N) \times \operatorname{Gr}_{\mathbf{e}_{2}}(M)$ by Theorem 1.2. $\left\langle\left(N_{1}, M_{1}\right)\right\rangle$ is a constructible subset and there exists a finite subset $R\left(\mathbf{e}_{1}, \mathbf{e}_{2}\right)$ of $\operatorname{Gr}_{\mathbf{e}_{1}}(N) \times G r_{\mathbf{e}_{2}}(M)$ such that

$$
\operatorname{Gr}_{\mathbf{e}_{1}}(N) \times \operatorname{Gr}_{\mathbf{e}_{2}}(M)=\bigsqcup_{\left(N_{1}, M_{1}\right) \in R\left(\mathbf{e}_{1}, \mathbf{e}_{2}\right)}\left\langle\left(N_{1}, M_{1}\right)\right\rangle .
$$

Hence, by Proposition 1.1, we have the following lemma.

Lemma 3.3. For fixed dimension vector $\mathbf{d}$, we have

$$
\begin{aligned}
& \sum_{\mathbf{e}_{1}, \mathbf{e}_{2} ; \mathbf{e}_{1}+\mathbf{e}_{2}=\mathbf{d}} \chi\left(\mathbb{P D F E}_{\mathbf{e}_{1}, \mathbf{e}_{2}}(N, \tau M)\right) \\
= & \sum_{\mathbf{e}_{1}, \mathbf{e}_{2} ; \mathbf{e}_{1}+\mathbf{e}_{2}=\mathbf{d}} \sum_{\left(N_{1}, M_{1}\right) \in R\left(\mathbf{e}_{1}, \mathbf{e}_{2}\right)} \chi\left(\left\langle\left(N_{1}, M_{1}\right)\right\rangle\right) \chi\left(\mathbb{P H o m}\left(N / N_{1}, \tau M_{1}\right)\right) .
\end{aligned}
$$

Now, we can compare $\left.\operatorname{DEF}_{\mathbf{e}_{1}, \mathbf{e}_{2}}^{\mathbf{d}_{1}, \mathbf{d}_{2}}(N, \tau M)\right)$ with $\left.\operatorname{DFE}_{\mathbf{e}_{1}, \mathbf{e}_{2}}(N, \tau M)\right)$. Let $\left(g, V_{1}, U_{1}\right)$ $\in D E F_{\mathbf{e}_{1}, \mathbf{e}_{2}}^{\mathbf{d}_{1}, \mathbf{d}_{2}}(N, \tau M)$. Then we have a long exact sequence

$$
0 \rightarrow V \rightarrow N \stackrel{g}{\rightarrow} \tau M \rightarrow \tau U \oplus I \rightarrow 0,
$$

where $V=\Upsilon_{0} \Upsilon^{2}(g)$ and $U=\phi^{-} \Omega_{0} \Omega^{2}(g)$. By definition, $\Upsilon_{0}^{-1}\left(V_{1}\right) \in \operatorname{Gr}_{\mathbf{e}_{1}}(N)$. By Proposition 2.1 and Corollary 2.2, we have a morphism of varieties $\operatorname{Gr}_{\mathbf{e}_{1}}(N) \rightarrow$ $\mathbb{E}_{\operatorname{dim} N-\mathbf{e}_{1}}$ sending any submodule $N_{1}$ to a $\mathbb{C} Q$-module isomorphic to the quotient module $N / N_{1}$. Let $U^{*}$ be the pullback of the following diagram:

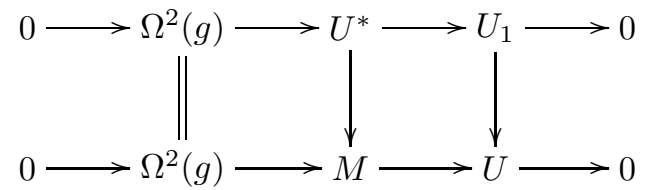

Note that $\Omega^{2}(g)=\operatorname{Im} g$. Then $U^{*} \in \operatorname{Gr}_{\operatorname{dim} M-\operatorname{dim} U+\operatorname{dim} U_{1}}(M)$, and we have the commutative diagram

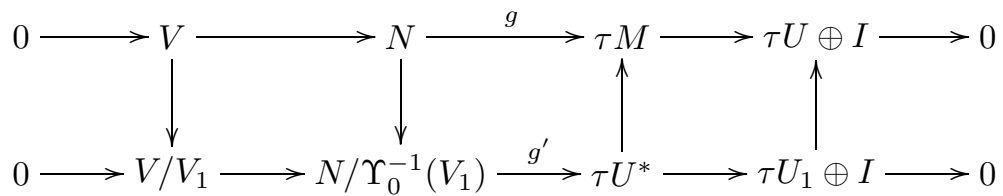


Hence, for fixed dimension vector $\mathbf{d}$, by Proposition 2.1. Corollary 2.2 and Proposition 2.5. we have a map whose restrictions to the strata are morphisms of varieties:

$$
\Gamma: \bigsqcup_{\substack{\mathbf{d}_{1}, \mathbf{d}_{2}, \mathbf{e}_{1}, \mathbf{e}_{2} ; \\ \mathbf{e}_{1}+\mathbf{e}_{2}+\operatorname{dim} M-\mathbf{d}_{2}=\mathbf{d}}} \operatorname{DEF}_{\mathbf{e}_{1}, \mathbf{e}_{2}}^{\mathbf{d}_{1}, \mathbf{d}_{2}}(N, \tau M) \rightarrow \underset{\mathbf{e}_{1}^{\prime}, \mathbf{e}_{2}^{\prime} ; \mathbf{e}_{1}^{\prime}+\mathbf{e}_{2}^{\prime}=\mathbf{d}}{\bigsqcup} \operatorname{DFE}_{\mathbf{e}_{1}^{\prime}, \mathbf{e}_{2}^{\prime}}(N, \tau M)
$$

mapping $\left(g, V_{1}, U_{1}\right)$ to $\left(V_{1}, U^{*}, g^{\prime}\right)$. Conversely, we have an inverse map whose restrictions to the strata are morphisms of varieties:

$$
\Gamma^{\prime}: \bigsqcup_{\mathbf{e}_{1}^{\prime}, \mathbf{e}_{2}^{\prime}} \operatorname{DFE}_{\mathbf{e}_{1}^{\prime}, \mathbf{e}_{2}^{\prime}}(N, \tau M) \rightarrow \underset{\substack{\mathbf{d}_{1}, \mathbf{d}_{2}, \mathbf{e}_{1}, \mathbf{e}_{2} ; \\ \mathbf{e}_{1}+\mathbf{e}_{2}+\mathbf{d i m} M-\mathbf{d}_{2}=\mathbf{d},}}{D^{2}} \operatorname{DEF}_{\mathbf{e}_{1}, \mathbf{e}_{2}}^{\mathbf{d}_{1}, \mathbf{d}_{2}}(N, \tau M) .
$$

The action of $\mathbb{C}^{*}$ induces a bijection whose restrictions to the strata are morphisms of varieties:

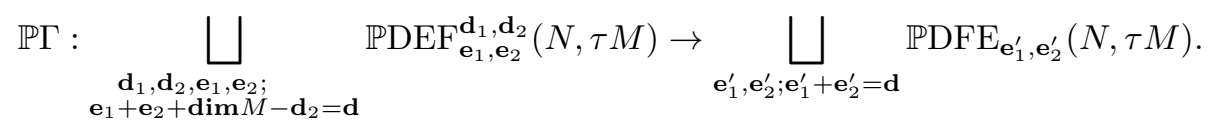

By Lemmas 3.2 and 3.3, the above bijection yields the following proposition referred to as the higher order associativity.

Proposition 3.4. For fixed dimension vector $\mathbf{d}$, we have

$$
\begin{aligned}
& \sum_{\substack{\mathbf{d}_{1}, \mathbf{d}_{2}, \mathbf{e}_{1}, \mathbf{e}_{2}, U, V, I ; \\
\mathbf{e}_{1}+\mathbf{e}_{2}+\mathbf{d i m} M-\mathbf{d}_{2}=\mathbf{d}, U \in S\left(\mathbf{d}_{2}\right), V \in S\left(\mathbf{d}_{1}\right)}} \chi\left(\mathbb{P H o m}(N, \tau M)_{\langle V\rangle \oplus\langle U\rangle \oplus I[-1]}\right) \chi\left(\operatorname{Gr}_{\mathbf{e}_{1}}(V)\right) \chi\left(\mathrm{Gr}_{\mathbf{e}_{2}}(U)\right) \\
& =\sum_{\substack{\mathbf{e}_{1}^{\prime}, \mathbf{e}_{2}^{\prime} ; \mathbf{e}_{1}^{\prime}+\mathbf{e}_{2}^{\prime}=\mathbf{d} \\
\left(N_{1}, M_{1}\right) \in R\left(\mathbf{e}_{1}^{\prime}, \mathbf{e}_{2}^{\prime}\right)}} \chi\left(\left\langle\left(N_{1}, M_{1}\right)\right\rangle\right) \chi\left(\mathbb{P H o m}\left(N / N_{1}, \tau M_{1}\right)\right) .
\end{aligned}
$$

\section{MAIN THEOREM AND PROOF}

4.1. The main theorem. We introduce some notation. For any $\mathbb{C} Q$-module $M$ and projective $\mathbb{C} Q$-module $P$, let $I=\operatorname{DHom}_{\mathbb{C} Q}(P, \mathbb{C} Q)$. By Corollary 1.4, we have the following finite partitions:

$$
\begin{aligned}
& \operatorname{Hom}(M, I)=\bigsqcup_{I^{\prime}, V \in S\left(\mathbf{d}_{1}\left(I^{\prime}\right)\right)} \operatorname{Hom}(M, I)_{\langle V\rangle \oplus I^{\prime}[-1]}, \\
& \operatorname{Hom}(P, M)=\bigsqcup_{P^{\prime}, U \in S\left(\mathbf{d}_{2}\left(P^{\prime}\right)\right)} \operatorname{Hom}(P, M)_{P^{\prime}[1] \oplus\langle U\rangle},
\end{aligned}
$$

where $\mathbf{d}_{1}\left(I^{\prime}\right)=\operatorname{dim} I+\operatorname{dim} I^{\prime}-\operatorname{dim} M, \mathbf{d}_{2}\left(P^{\prime}\right)=\operatorname{dim} P+\operatorname{dim} P^{\prime}-\operatorname{dim} M, \mathbb{E}_{\mathbf{d}_{1}}=$ $\bigsqcup_{V \in S\left(\mathbf{d}_{1}\right)}\langle V\rangle, \mathbb{E}_{\mathbf{d}_{2}}=\bigsqcup_{U \in S\left(\mathbf{d}_{2}\right)}\langle U\rangle, \operatorname{Hom}(M, I)_{\langle V\rangle \oplus I^{\prime}[-1]}=\{f \in \operatorname{Hom}(P, M) \mid$ $\Upsilon_{0} \Upsilon^{2}(f)=V^{\prime}, \Omega_{0} \Omega^{2}(f)=I^{\prime}$ for some $\left.V^{\prime} \in\langle V\rangle\right\}$, and $\operatorname{Hom}(P, M)_{P^{\prime}[1] \oplus\langle U\rangle}=$ $\left\{g \in \operatorname{Hom}(P, M) \mid \Upsilon_{0} \Upsilon^{2}(g)=P^{\prime}, \Omega_{0} \Omega^{2}(g)=U^{\prime}\right.$ for some $\left.U^{\prime} \in\langle U\rangle\right\}$. Note that $\Upsilon_{0} \Upsilon^{2}(f) \cong \operatorname{ker} f$ and $\Omega_{0} \Omega^{2}(f) \cong \operatorname{coker} f$.

The following theorem generalizes the cluster multiplication theorem for finite type in [3] and affine type in [14] and is referred to as the cluster multiplication theorem for arbitrary type. It was proved in [31] using different techniques. 
Theorem 4.1. Let $Q$ be an acyclic quiver. Then

(1) for any $\mathbb{C} Q$-modules $M, N$ such that $M$ contains no projective summand, we have

$$
\begin{aligned}
& \operatorname{dim}_{\mathbb{C}} \operatorname{Ext}_{\mathbb{C Q}}^{1}(M, N) X_{M} X_{N}=\sum_{L \in S(\mathbf{e})} \chi\left(\mathbb{P E x t}_{\mathbb{C} Q}^{1}(M, N)_{\langle L\rangle}\right) X_{L} \\
& +\sum_{I, \mathbf{d}_{1}, \mathbf{d}_{2}} \sum_{V \in S\left(\mathbf{d}_{1}\right), U \in S\left(\mathbf{d}_{2}\right)} \chi\left(\mathbb{P H o m} \mathbb{C Q}(N, \tau M)_{\langle V\rangle \oplus\langle U\rangle \oplus I[-1]}\right) X_{V \oplus U \oplus I-1]},
\end{aligned}
$$

where $\mathbf{e}=\operatorname{dim} M+\operatorname{dim} N$ and

(2) for any $\mathbb{C} Q$-module $M$ and projective $\mathbb{C} Q$-module $P$, we have

$$
\begin{aligned}
& \operatorname{dim}_{\mathbb{C}} \operatorname{Hom}_{\mathbb{C Q}}(P, M) X_{M} X_{P[1]}=\sum_{I^{\prime}, V \in S\left(\mathbf{d}_{1}\left(I^{\prime}\right)\right)} \chi\left(\mathbb{P H o m}_{\mathbb{C Q}}(M, I)_{\langle V\rangle \oplus I^{\prime}[-1]}\right) X_{V \oplus I^{\prime}[-1]} \\
& +\sum_{P^{\prime}, U \in S\left(\mathbf{d}_{2}\left(P^{\prime}\right)\right)} \chi\left(\mathbb{P H o m}_{\mathbb{C} Q}(P, M)_{P^{\prime}[1] \oplus\langle U\rangle}\right) X_{P^{\prime}[1] \oplus U},
\end{aligned}
$$

where $I=\operatorname{DHom}_{\mathbb{C Q}}(P, \mathbb{C Q}), I^{\prime}$ is injective, and $P^{\prime}$ is projective.

Proof. We set

$$
\Sigma_{2}:=\sum_{I, \mathbf{d}_{1}, \mathbf{d}_{2}, V \in S\left(\mathbf{d}_{1}\right), U \in S\left(\mathbf{d}_{2}\right)} \chi\left(\mathbb{P H o m} \operatorname{CQ}_{\mathbb{Q}}(N, \tau M)_{\langle V\rangle \oplus\langle U\rangle \oplus I-1]}\right) X_{U} X_{V} x^{\text {dimsoc } I} .
$$

By the definition of $X_{M}$, the sum $\Sigma_{2}$ is equal to

$$
\begin{gathered}
\sum_{I, \mathbf{d}_{1}, \mathbf{d}_{2}, \mathbf{e}_{1}, \mathbf{e}_{2}, V \in S\left(\mathbf{d}_{1}\right), U \in S\left(\mathbf{d}_{2}\right)} \chi\left(\mathbb{P H o m}_{\mathbb{C Q}}(N, \tau M)_{\langle V\rangle \oplus\langle U\rangle \oplus I[-1]}\right) \\
\cdot \chi\left(\operatorname{Gr}_{\mathbf{e}_{1}}(V)\right) \chi\left(\operatorname{Gr}_{\mathbf{e}_{2}}(U)\right) x^{\left(\mathbf{e}_{1}+\mathbf{e}_{2}\right) R+\left(\operatorname{dim} V-\mathbf{e}_{1}+\operatorname{dim} U-\mathbf{e}_{2}\right) R^{t r}-(\operatorname{dim} V+\operatorname{dim} U)+\operatorname{dimsoc} I} .
\end{gathered}
$$

By Lemma 1.5, we have

$$
\begin{aligned}
& (\operatorname{dim} V+\operatorname{dim} U) R^{t r}-(\operatorname{dim} V+\operatorname{dim} U)+\operatorname{dimsoc} I \\
& \quad=(\operatorname{dim} V+\operatorname{dim} U) R^{t r}-(\operatorname{dim} V+\operatorname{dim} U)+\operatorname{dim} I\left(1-R^{t r}\right) \\
& \quad=(\operatorname{dim} \tau U+\operatorname{dim} I-\operatorname{dim} V)-(\operatorname{dim} \tau U+\operatorname{dim} I-\operatorname{dim} V) R^{t r}+\operatorname{dim} U\left(R^{t r}-R\right) \\
& \quad=(\operatorname{dim} \tau M-\operatorname{dim} N)-(\operatorname{dim} \tau M-\operatorname{dim} N) R^{t r}+\operatorname{dim} U\left(R^{t r}-R\right) \\
& \quad=\operatorname{dim} \tau M\left(1-R^{t r}\right)-\operatorname{dim} N+\operatorname{dim} N R^{t r}+\operatorname{dim} U\left(R^{t r}-R\right) \\
& \quad=(\operatorname{dim} M-\operatorname{dim} U) R+(\operatorname{dim} N+\operatorname{dim} U) R^{t r}-(\operatorname{dim} M+\operatorname{dim} N) .
\end{aligned}
$$

Then, the exponent of $x$ is

$\left(\mathbf{e}_{1}+\mathbf{e}_{2}+\operatorname{dim} M-\operatorname{dim} U\right) R+\left(\operatorname{dim} N+\operatorname{dim} U-\mathbf{e}_{1}-\mathbf{e}_{2}\right) R^{t r}-(\operatorname{dim} M+\operatorname{dim} N)$.

Hence, we have

$$
\begin{aligned}
\Sigma_{2}= & \sum_{\mathbf{d}} \sum_{\substack{I, \mathbf{d}_{1}, \mathbf{d}_{2}, \mathbf{e}_{1}, \mathbf{e}_{2} ; \\
\mathbf{e}_{1}+\mathbf{e}_{2}+\mathbf{d i m} M-\mathbf{d}_{2}=\mathbf{d}}} \sum_{V \in S\left(\mathbf{d}_{1}\right), U \in S\left(\mathbf{d}_{2}\right)} \chi\left(\mathbb{P H o m}_{\mathbb{C Q}}(N, \tau M)_{\langle V\rangle \oplus\langle U\rangle \oplus I[-1]}\right) \\
& \cdot \chi\left(\operatorname{Gr}_{\mathbf{e}_{1}}(V)\right) \chi\left(\operatorname{Gr}_{\mathbf{e}_{2}}(U)\right) x^{\mathbf{d} R+(\mathbf{e}-\mathbf{d}) R^{t r}-\mathbf{e}}
\end{aligned}
$$


where $\mathbf{e}=\operatorname{dim} M+\operatorname{dim} N$. Using Proposition 3.4, we obtain the fact that $\Sigma_{2}$ is equal to

$$
\begin{aligned}
\sum_{\mathbf{d}} \sum_{\mathbf{e}_{1}^{\prime}, \mathbf{e}_{2}^{\prime} ; \mathbf{e}_{1}^{\prime}+\mathbf{e}_{2}^{\prime}=\mathbf{d}\left(N_{1}, M_{1}\right) \in R\left(\mathbf{e}_{1}^{\prime}, \mathbf{e}_{2}^{\prime}\right)} \chi\left(\left\langle\left(N_{1}, M_{1}\right)\right\rangle\right) \\
\cdot \chi\left(\mathbb{P H o m}_{\mathbb{C Q}}\left(N / N_{1}, \tau M_{1}\right)\right) x^{\mathbf{d} R+(\mathbf{e}-\mathbf{d}) R^{t r}-\mathbf{e}} .
\end{aligned}
$$

Now we set

$$
\Sigma_{1}:=\sum_{L \in S(\mathbf{e})} \chi\left(\operatorname{PExt}_{\mathbb{C Q}}^{1}(M, N)_{\langle L\rangle}\right) X_{L}
$$

and

$$
\operatorname{EF}_{\mathbf{d}}(M, N):=\left\{\left(\varepsilon, L_{1}\right) \mid \varepsilon \in \operatorname{Ext}_{\mathbb{C} Q}^{1}(M, N)_{L} \backslash\{0\}, L_{1} \in \operatorname{Gr}_{\mathbf{d}}(L)\right\} .
$$

As a vector space, $L=M \oplus N$. Define

$$
\text { t. }(m, n)=(m, t . n)
$$

for any $(m, n) \in M \oplus N$ and $t \in \mathbb{C}^{*}$. This induces the action of $\mathbb{C}^{*}$ on $L_{1}$. So we have an $\mathbb{C}^{*}$-action on $\operatorname{EF}_{\mathbf{d}}(M, N)([12])$. By the discussion in Section 3.2, the $\mathbb{C}^{*}$-action induces the geometric quotient $\operatorname{PEF}_{\mathbf{d}}(M, N)$. The projection

$$
\mathbb{P E F}_{\mathbf{d}}(M, N) \rightarrow \mathbb{P E x t}_{\mathbb{C} Q}^{1}(M, N)
$$

has the fibre $\left\{\left(\mathbb{P} \varepsilon, L_{1}\right) \mid L_{1} \in \operatorname{Gr}_{\mathbf{d}}(L)\right\}$ for any $\mathbb{P} \varepsilon \in \mathbb{P E x t}_{\mathbb{C} Q}^{1}(M, N)_{L}$. By Theorem 1.1 and Corollary 1.4 this implies

$$
\chi\left(\mathbb{P E F}_{\mathbf{d}}(M, N)\right)=\sum_{L \in S(\mathbf{e})} \chi\left(\mathbb{P E x t}_{\mathbb{C Q}}^{1}(M, N)_{\langle L\rangle}\right) \chi\left(\operatorname{Gr}_{\mathbf{d}}(L)\right)
$$

and

$$
\Sigma_{1}=\sum_{\mathbf{d}} \chi\left(\mathbb{P E F}_{\mathbf{d}}(M, N)\right) x^{\mathbf{d} R+(\mathbf{e}-\mathbf{d}) R^{t r}-\mathbf{e}} .
$$

On the other hand, we have a natural morphism

$$
\Psi: \mathrm{EF}_{\mathbf{d}}(M, N) \rightarrow \underset{\mathbf{e}_{1}^{\prime}, \mathbf{e}_{2}^{\prime} ; \mathbf{e}_{1}^{\prime}+\mathbf{e}_{2}^{\prime}=\mathbf{d}}{ } \operatorname{Gr}_{\mathbf{e}_{1}^{\prime}}(M) \times \operatorname{Gr}_{\mathbf{e}_{2}^{\prime}}(N)
$$

mapping $\left(\varepsilon=[(f, g)], L_{1}\right)$ to $\left(g\left(L_{1}\right), f^{-1}\left(L_{1}\right)\right)$. Here $\varepsilon$ is the equivalence class of the exact sequence

$$
0 \rightarrow N \stackrel{f}{\rightarrow} L \stackrel{g}{\rightarrow} M \rightarrow 0 .
$$

The morphism $\Psi$ induces a morphism

$$
\mathbb{P} \Psi: \mathbb{P E F}(M, N):=\bigsqcup_{\mathbf{d}} \operatorname{PEF}_{\mathbf{d}}(M, N) \rightarrow \bigsqcup_{\mathbf{e}_{1}^{\prime}, \mathbf{e}_{2}^{\prime}} \operatorname{Gr}_{\mathbf{e}_{1}^{\prime}}(M) \times \operatorname{Gr}_{\mathbf{e}_{2}^{\prime}}(N) .
$$

Let's compute the fibre for any $\left(M_{1}, N_{1}\right) \in \operatorname{Gr}_{\mathbf{e}_{1}^{\prime}}(M) \times \operatorname{Gr}_{\mathbf{e}_{2}^{\prime}}(N)$. Consider the map

$$
\beta^{\prime}: \operatorname{Ext}^{1}(M, N) \oplus \operatorname{Ext}_{\mathbb{C} Q}^{1}\left(M_{1}, N_{1}\right) \rightarrow \operatorname{Ext}_{\mathbb{C} Q}^{1}\left(M_{1}, N\right)
$$

sending $\left(\varepsilon, \varepsilon^{\prime}\right)$ to $\varepsilon_{M_{1}}-\varepsilon_{N}^{\prime}$, where $\varepsilon_{M_{1}}$ and $\varepsilon_{N}^{\prime}$ are induced by including $M_{1} \subseteq M$ and $N_{1} \subseteq N$, respectively, as follows:

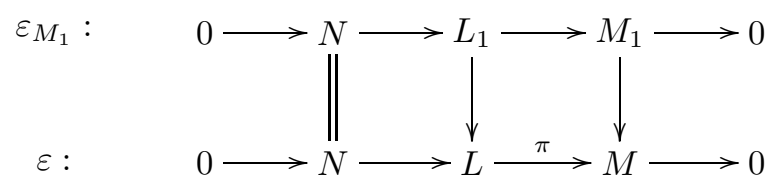


where $L_{1}$ is the pullback, and

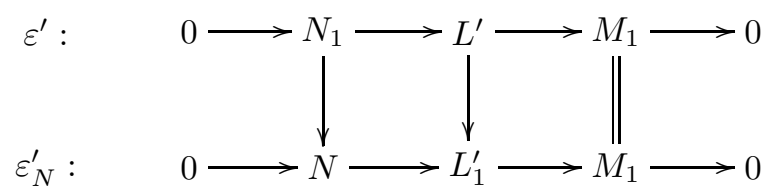

where $L_{1}^{\prime}$ is the pushout. It is clear that $\varepsilon, \varepsilon^{\prime}$ and $M_{1}, N_{1}$ induce the inclusions $L_{1} \subseteq L$ and $L^{\prime} \subseteq L_{1}^{\prime}$. Considering the map

$$
p_{0}: \operatorname{Ext}_{\mathbb{C} Q}^{1}(M, N) \oplus \operatorname{Ext}_{\mathbb{C} Q}^{1}\left(M_{1}, N_{1}\right) \rightarrow \operatorname{Ext}_{\mathbb{C} Q}^{1}(M, N),
$$

we have ([12, Lemma 2.4.2])

$$
(\mathbb{P} \Psi)^{-1}\left(M_{1}, N_{1}\right)=\left\{\left(\mathbb{P} \varepsilon, L^{\prime}\right) \mid \mathbb{P} \varepsilon \in \mathbb{P}\left(p_{0}\left(\operatorname{ker} \beta^{\prime}\right)\right), L^{\prime} \in F\left(\varepsilon, M_{1}, N_{1}\right)\right\},
$$

where $F\left(\varepsilon, M_{1}, N_{1}\right)=\left\{L^{\prime} \subseteq L \mid \pi\left(L^{\prime}\right)=M_{1}, L^{\prime} \cap N=N_{1}\right\}$ is isomorphic to the affine space $\operatorname{Hom}\left(M_{1}, N / N_{1}\right)$ or an empty set (14, Lemma 7]; see also [12, Lemma 3.3.1] for a similar discussion). By the 2-Calabi-Yau property (Auslander-Reiten formula) $\operatorname{Ext}^{1}(M, N) \simeq D \operatorname{Hom}(N, \tau M)$, we can consider the dual of $\beta^{\prime}$ which is

$$
\beta: \operatorname{Hom}\left(N, \tau M_{1}\right) \rightarrow \operatorname{Hom}(N, \tau M) \oplus \operatorname{Hom}\left(N_{1}, \tau M_{1}\right) .
$$

By using the knowledge of bilinear form and orthogonality, we know that as a vector space,

$$
\left(p_{0}\left(\operatorname{ker} \beta^{\prime}\right)\right)^{\perp}=\operatorname{Im} \beta \cap \operatorname{Hom}(N, \tau M) \simeq \operatorname{Hom}\left(N / N_{1}, \tau M_{1}\right) .
$$

Note that if $F\left(\varepsilon, M_{1}, N_{1}\right)$ is an empty set, then $\mathbb{P}\left(p_{0}\left(\operatorname{ker} \beta^{\prime}\right)\right)$ is an empty set. In this case, $\operatorname{dim}_{\mathbb{C}} \operatorname{Ext}^{1}(M, N)=\chi\left(\mathbb{P H o m}\left(N / N_{1}, \tau M_{1}\right)\right)$. Hence, we obtain

$$
\chi\left((\mathbb{P} \Psi)^{-1}\left(M_{1}, N_{1}\right)\right)=\operatorname{dim}_{\mathbb{C}} \operatorname{Ext}^{1}(M, N)-\chi\left(\mathbb{P} \operatorname{Hom}\left(N / N_{1}, \tau M_{1}\right)\right) .
$$

Now, using the partitions as in Proposition 3.4 we know

$$
\operatorname{Gr}_{\mathbf{e}_{1}^{\prime}}(M) \times \operatorname{Gr}_{\mathbf{e}_{2}^{\prime}}(N)=\bigsqcup_{\left(N_{1}, M_{1}\right) \in R\left(\mathbf{e}_{1}^{\prime}, \mathbf{e}_{2}^{\prime}\right)}\left\langle\left(N_{1}, M_{1}\right)\right\rangle .
$$

Hence, according the Euler characteristic of the fibres in (4.1) and Proposition 1.1. we obtain the fact that $\Sigma_{1}$ is equal to

$$
\begin{aligned}
& \sum_{\mathbf{d}} \sum_{\mathbf{e}_{1}^{\prime}, \mathbf{e}_{2}^{\prime} ; \mathbf{e}_{1}^{\prime}+\mathbf{e}_{2}^{\prime}=\mathbf{d}} \sum_{\left(N_{1}, M_{1}\right) \in R\left(\mathbf{e}_{1}^{\prime}, \mathbf{e}_{2}^{\prime}\right)} \\
& \cdot \chi\left(\left\langle\left(N_{1}, M_{1}\right)\right\rangle\right)\left(\operatorname{dim}_{\mathbb{C}} \operatorname{Ext}^{1}(M, N)-\chi\left(\mathbb{P H o m} \mathbb{C Q}\left(N / N_{1}, \tau M_{1}\right)\right)\right) x^{\mathbf{d} R+(\mathbf{e}-\mathbf{d}) R^{t r}-\mathbf{e}} .
\end{aligned}
$$

Hence,

$$
\Sigma_{1}+\Sigma_{2}=\operatorname{dim}_{\mathbb{C}} \operatorname{Ext}^{1}(M, N) X_{M} X_{N} .
$$

We complete the proof of the first assertion of the theorem. As for the second part, we set

$$
\begin{aligned}
T_{1}= & \sum_{I^{\prime}, \mathbf{e}_{1}, V ; V \in S\left(\mathbf{d}_{1}\left(I^{\prime}\right)\right)} \chi\left(\mathbb{P H o m}_{\mathbb{C Q}}(M, I)_{\langle V\rangle \oplus I^{\prime}[-1]}\right) \chi\left(G r_{\mathbf{e}_{1}}(V)\right) \\
& \cdot x^{\mathbf{e}_{1} R+\left(\mathbf{d}_{1}\left(I^{\prime}\right)-\mathbf{e}_{1}\right) R^{t r}-\mathbf{d}_{1}\left(I^{\prime}\right)+\operatorname{dimsoc} I^{\prime}}
\end{aligned}
$$


and

$$
\begin{aligned}
T_{2}= & \sum_{P^{\prime}, \mathbf{e}_{2}, U ; U \in S\left(\mathbf{d}_{2}\left(P^{\prime}\right)\right)} \chi\left(\mathbb{P H o m}_{\mathbb{C Q}}(P, M)_{P^{\prime}[1] \oplus\langle U\rangle}\right) \chi\left(\operatorname{Gr}_{\mathbf{e}_{2}}(V)\right) \\
& \cdot x^{\mathbf{e}_{2} R+\left(\mathbf{d}_{2}\left(P^{\prime}\right)-\mathbf{e}_{2}\right) R^{t r}-\mathbf{d}_{2}\left(P^{\prime}\right)+\operatorname{dim} t o p\left(P^{\prime}\right)} .
\end{aligned}
$$

By a similar argument as in Corollary 1.4, there is a finite subset $R\left(\mathbf{e}_{1}\right)$ of $\operatorname{Gr}_{\mathbf{e}_{1}}(M)$ such that the partition

$$
\operatorname{Gr}_{\mathbf{e}_{1}}(M)=\bigsqcup_{M_{1} \in R\left(\mathbf{e}_{1}\right)}\left\{M_{1}\right\}
$$

where $\left\{M_{1}\right\}=\left\{W \in \operatorname{Gr}_{\mathbf{e}_{1}}(M) \mid \chi(\mathbb{P H o m}(M / W, I))=\chi\left(\mathbb{P H o m}\left(M / M_{1}, I\right)\right)\right\}$ is a constructible subset of $\mathrm{Gr}_{\mathbf{e}_{1}}(M)$. Note that

$$
\left\{M_{1}\right\}=\left\{W \in \operatorname{Gr}_{\mathbf{e}_{1}}(M) \mid \chi(\mathbb{P H o m}(P, W))=\chi\left(\mathbb{P H o m}\left(P, M_{1}\right)\right)\right\} .
$$

By using Proposition 1.1, we obtain that $T_{1}$ is equal to

$$
\sum_{\mathbf{e}_{1}} \sum_{M_{1} \in R\left(\mathbf{e}_{1}\right)} \chi\left(\left\{M_{1}\right\}\right) \cdot \chi\left(\mathbb{P H o m}\left(M / M_{1}, I\right)\right) x^{\mathbf{e}_{1} R+\left(\operatorname{dim} M-\mathbf{e}_{1}\right) R^{t r}-\underline{d}_{M}+\operatorname{dimsoc}(I)}
$$

and $T_{2}$ is equal to

$$
\sum_{\mathbf{e}_{1}} \sum_{M_{1} \in R\left(\mathbf{e}_{1}\right)} \chi\left(\left\{M_{1}\right\}\right) \cdot \chi\left(\mathbb{P H o m}\left(P, M_{1}\right)\right) x^{\mathbf{e}_{1} R+\left(\operatorname{dim} M-\mathbf{e}_{1}\right) R^{t r}-\operatorname{dim} M+\operatorname{dim} t o p(P)} .
$$

Since $\operatorname{dimsoc}(I)=\operatorname{dim} t o p(P)$ and

$$
\chi\left(\mathbb{P H o m}\left(P, M_{1}\right)\right)+\chi\left(\mathbb{P H o m}\left(M / M_{1}, I\right)\right)=\chi(\mathbb{P H o m}(P, M)),
$$

we have

$$
\begin{aligned}
T_{1} & +T_{2}=\operatorname{dim}_{\mathbb{C}} \operatorname{Hom}(P, M) \sum_{\mathbf{e}} \chi\left(\operatorname{Gr}_{\mathbf{e}}(M)\right) x^{\mathbf{e} R+(\operatorname{dim} M-\mathbf{e}) R^{t r}-\operatorname{dim} M+\operatorname{dim} t o p(P)} \\
& =\operatorname{dim}_{\mathbb{C}} \operatorname{Hom}_{\mathbb{C} Q}(P, M) X_{M} x^{\operatorname{dim} t o p(P)}
\end{aligned}
$$

Remark 4.2. The proof of Theorem4.1 only involves the Auslander-Reiten formula and the higher order associativity. It inspires us to look for an analog of the cluster multiplication theorem for hereditary categories with Serre duality. The simplest case is as follows: if $Q$ is a Kronecker quiver, then we know $\mathcal{D}^{b}(Q)$ is derived equivalent to $\mathcal{D}^{b}\left(\operatorname{coh} \mathbb{P}^{1}\right)$. We expect that the present approach can help us find a cluster multiplication formula for $\operatorname{coh} \mathbb{P}^{1}$. One of the difficulties is to replace module varieties with stacks to rewrite the results in this paper as done in [16] and 27]. It will be interesting to compare these cluster multiplication theorems.

Remark 4.3. In Theorem 4.1 the condition that $M$ contains no projective summands is not essential. Let $M^{\prime}=M \oplus P$ with the maximal projective summand $P$. Then we multiply two sides of the first equation in Theorem 4.1 by $X_{P}$ to obtain the equation involving $X_{M^{\prime}} X_{N}$.

Now we consider the particular case where $M$ is a non-projective indecomposable $\mathbb{C} Q$-module and $N=\tau M$. By the Auslander-Reiten formula, there is an isomorphism of $\operatorname{End}_{\mathbb{C} Q}(M)^{o p}$-modules: $\operatorname{Ext}_{\mathbb{C} Q}^{1}(M, \tau M) \cong D \operatorname{End}_{\mathbb{C} Q}(\tau M)$. It induces the isomorphisms

$$
\operatorname{socExt}_{\mathbb{C} Q}^{1}(M, \tau M) \cong D\left(\operatorname{End}_{\mathbb{C} Q}(\tau M) / \operatorname{radEnd}_{\mathbb{C} Q}(\tau M)\right),
$$


where socExt ${ }_{\mathbb{C} Q}^{1}(M, \tau M)$ is the socle of $\operatorname{Ext}_{\mathbb{C} Q}^{1}(M, \tau M)$ as an $\operatorname{End}_{\mathbb{C} Q}(M)^{o p}$-module, and

$$
\operatorname{Ext}_{\mathbb{C Q}}^{1}(M, \tau M) / \operatorname{socExt}_{\mathbb{C Q}}^{1}(M, \tau M) \cong D\left(\operatorname{radEnd}_{\mathbb{C} Q}(\tau M)\right) .
$$

The equations (4.2) and (4.3) can be viewed as variants of the 2-Calabi-Yau property (the Auslander-Reiten formula). An extension $\varepsilon \in \operatorname{Ext}_{\mathbb{C} Q}^{1}(M, \tau M)$ is an AuslanderReiten sequence if and only if $\varepsilon \in \operatorname{socExt}_{\mathbb{C} Q}^{1}(M, \tau M)$. We denote by $L_{0}$ the middle term of $\varepsilon$. In the proof of Theorem 4.1, we substitute socExt $\operatorname{Cx}_{\mathbb{C} Q}^{1}(M, \tau M)$ or $\operatorname{Ext}_{\mathbb{C} Q}^{1}(M, \tau M) / \operatorname{socExt}_{\mathbb{C} Q}^{1}(M, \tau M)$ for $\operatorname{Ext}_{\mathbb{C} Q}^{1}(M, \tau M)$ and the above variants (4.2) or (4.3) for the Auslander-Reiten formula. Then we obtain the following result (see 2] or Lemma 7 in [14] for different proofs).

Proposition 4.4. Let $Q$ be an acyclic quiver and $M$ be a non-projective indecomposable $\mathbb{C} Q$-module. Then

$$
\begin{aligned}
\operatorname{dim}_{\mathbb{C}} \operatorname{Ext}_{\mathbb{C} Q}^{1}(M, \tau M) / \operatorname{socExt}_{\mathbb{C Q}}^{1}(M, \tau M) X_{M} X_{\tau M} \\
=\sum_{L \neq L_{0} \in S(\mathbf{e})} \chi\left(\mathbb{P E x t}_{\mathbb{C} Q}^{1}(M, \tau M)_{\langle L\rangle}\right) X_{L} \\
\left.\quad+\sum_{I, \mathbf{d}_{1}, \mathbf{d}_{2}} \sum_{V \in S\left(\mathbf{d}_{1}\right), U \in S\left(\mathbf{d}_{2}\right)} \chi\left(\operatorname{PradEnd}_{\mathbb{C} Q}(\tau M)\langle V\rangle \oplus\langle U\rangle \oplus I-1\right]\right) X_{U} X_{V} x^{\text {dimsocI }}
\end{aligned}
$$

and

$$
X_{M} X_{\tau M}=1+X_{L_{0}}
$$

where $\mathbf{e}=\operatorname{dim} M+\operatorname{dim} \tau M$ and $\operatorname{PradEnd}_{\mathbb{C} Q}(\tau M)$ is the quotient of $\operatorname{radEnd}_{\mathbb{C} Q}(\tau M)$ under the free action of $\mathbb{C}^{*}$ and $L_{0}$ is the middle term of the Auslander-Reiten sequence ending in $M$.

Proof. We only need to prove the second equation. It is equivalent to prove that $\operatorname{dim}_{\mathbb{C}} \operatorname{socExt}_{\mathbb{C} Q}^{1}(M, \tau M) X_{M} X_{\tau M}$ is equal to

$$
\chi\left(\mathbb{P E x t}_{\mathbb{C} Q}^{1}(M, \tau M)_{L_{0}}\right) X_{L_{0}}+\chi\left(\mathbb{P}\left(\operatorname{End}_{\mathbb{C} Q}(\tau M) / \operatorname{radEnd}_{\mathbb{C} Q}(\tau M)\right)\right) .
$$

We use the notation in the proof of Theorem 4.1 and set

$$
\Sigma_{1}:=\chi\left(\mathbb{P E x t}_{\mathbb{C} Q}^{1}(M, \tau M)_{L_{0}}\right) X_{L_{0}}
$$

and

$$
\operatorname{EF}_{\mathbf{d}}(M, \tau M)=\left\{\left(\varepsilon, L_{1}\right) \mid \varepsilon \in \operatorname{Ext}_{\mathbb{C Q}}^{1}(M, N)_{L_{0}}, L_{1} \in \operatorname{Gr}_{\mathbf{d}}\left(L_{0}\right)\right\} .
$$

The $\mathbb{C}^{*}$-action induces the geometric quotient $\mathbb{P E F}_{\mathbf{d}}(M, \tau M)$. We have

$$
\Sigma_{1}=\sum_{\mathbf{d}} \chi\left(\mathbb{P E F}_{\mathbf{d}}(M, \tau M)\right) x^{\mathbf{d} R+(\mathbf{e}-\mathbf{d}) R^{t r}-\mathbf{e}}
$$

and a morphism

$$
\mathbb{P} \Psi: \mathbb{P E F}(M, \tau M):=\bigsqcup_{\mathbf{d}} \operatorname{PEF}_{\mathbf{d}}(M, \tau M) \rightarrow \bigsqcup_{\mathbf{e}_{1}^{\prime}, \mathbf{e}_{2}^{\prime}} \operatorname{Gr}_{\mathbf{e}_{1}^{\prime}}(M) \times \operatorname{Gr}_{\mathbf{e}_{2}^{\prime}}(\tau M) .
$$

For any $\left(M_{1}, N_{1}\right) \in \operatorname{Gr}_{\mathbf{e}_{1}^{\prime}}(M) \times \operatorname{Gr}_{\mathbf{e}_{2}^{\prime}}(\tau M)$, we consider the map

$$
\beta^{\prime}: \operatorname{socExt}^{1}(M, \tau M) \oplus \operatorname{Ext}_{\mathbb{C} Q}^{1}\left(M_{1}, N_{1}\right) \rightarrow \operatorname{Ext}_{\mathbb{C} Q}^{1}\left(M_{1}, \tau M\right)
$$

and the map

$$
p_{0}: \operatorname{socExt}{ }_{\mathbb{C} Q}^{1}(M, \tau M) \oplus \operatorname{Ext}_{\mathbb{C} Q}^{1}\left(M_{1}, N_{1}\right) \rightarrow \operatorname{socExt}_{\mathbb{C} Q}^{1}(M, \tau M) .
$$


Then as in the proof of Theorem 4.1 we have

$$
(\mathbb{P} \Psi)^{-1}\left(M_{1}, N_{1}\right)=\left\{\left(\mathbb{P} \varepsilon, L_{1}\right) \mid \mathbb{P} \varepsilon \in \mathbb{P}\left(p_{0}\left(\operatorname{ker} \beta^{\prime}\right)\right), L_{1} \in F\left(\varepsilon, M_{1}, N_{1}\right)\right\},
$$

where $F\left(\varepsilon, M_{1}, N_{1}\right)=\left\{L_{1} \subseteq L \mid \pi\left(L_{1}\right)=M_{1}, L_{1} \cap N=N_{1}\right\}$ is isomorphic to the affine space $\operatorname{Hom}\left(M_{1}, N / N_{1}\right)$ or an empty set. By using the variant (4.2) of the Auslander-Reiten formula $\operatorname{socExt}{ }^{1}(M, N) \simeq D(\operatorname{End}(\tau M) / \operatorname{radEnd}(M))$, we can consider the dual of $\beta^{\prime}$ :

$$
\beta: \operatorname{Hom}\left(\tau M, \tau M_{1}\right) \rightarrow \operatorname{End}(\tau M) / \operatorname{radEnd}(M) \oplus \operatorname{Hom}\left(N_{1}, \tau M_{1}\right) .
$$

Then

$$
\left(p_{0}\left(\operatorname{ker} \beta^{\prime}\right)\right)^{\perp}=\operatorname{Im} \beta \cap \operatorname{End}(\tau M) / \operatorname{radEnd}(M),
$$

which vanishes unless $N_{1}=0$ and $M_{1}=M$. Hence, we obtain

$$
\chi\left((\mathbb{P} \Psi)^{-1}\left(M_{1}, N_{1}\right)\right)=\left\{\begin{array}{cc}
\operatorname{dim}_{\mathbb{C} \operatorname{soc} \operatorname{Ext}^{1}}(M, N), & \text { if } N_{1} \neq 0 \text { or } M_{1} \neq M, \\
0, & \text { otherwise }
\end{array}\right.
$$

This implies equation (4.4).

4.2. An example. Let us illustrate Theorem 4.1 and Proposition 4.4 by the following example. Let $Q$ be the Kronecker quiver $1 \Longrightarrow 2$. Let $S_{1}$ and $S_{2}$ be the simple modules associated to vertices 1 and 2 , respectively. Hence,

$$
R=\left(\begin{array}{cc}
0 & 2 \\
0 & 0
\end{array}\right) \quad \text { and } \quad R^{t r}=\left(\begin{array}{cc}
0 & 0 \\
2 & 0
\end{array}\right)
$$

and

$$
\begin{aligned}
& x_{0}:=X_{S_{2}}=x \underline{\operatorname{dim}} S_{2} R^{t r}-\underline{\operatorname{dim}} S_{2}+x \underline{\operatorname{dim}} S_{2} R-\underline{\operatorname{dim}} S_{2}=x_{2}^{-1}\left(1+x_{1}^{2}\right), \\
& x_{3}:=X_{S_{1}}=x \underline{\operatorname{dim}} S_{1} R^{t r}-\underline{\operatorname{dim}} S_{1}+x \underline{\operatorname{dim}} S_{1} R-\underline{\operatorname{dim}} S_{1}=x_{1}^{-1}\left(1+x_{2}^{2}\right) .
\end{aligned}
$$

For $\lambda \in \mathbb{P}^{1}(\mathbb{C})$, let $u_{\lambda}$ be the regular representation $\mathbb{C} \underset{\lambda}{\stackrel{1}{\longrightarrow}} \mathbb{C}$. By definition, $X_{u_{\lambda}}=x^{(1,1) R^{\prime}-(1,1)}+x^{(1,1) R-(1,1)}+x^{(0,1) R+(1,0) R^{\prime}-(1,1)}=x_{1} x_{2}^{-1}+x_{1}^{-1} x_{2}+x_{1}^{-1} x_{2}^{-1}$. Similarly, let $u_{\lambda(n)}(n \geq 1)$ be the unique indecomposable regular $\mathbb{C} Q$-module with socle $u_{\lambda}$ and length $n$. In particular, $u_{\lambda(1)}=u_{\lambda}$. Then $\operatorname{dim}_{\mathbb{C}} \operatorname{Ext}^{1}\left(u_{\lambda}, u_{\lambda(n)}\right)=1$, and for any $f \neq 0 \in \operatorname{Hom}\left(u_{\lambda(n)}, \tau u_{\lambda}\right)$ we have a short exact sequence

$$
0 \rightarrow u_{\lambda(n-1)} \rightarrow u_{\lambda(n)} \stackrel{f}{\rightarrow} \tau u_{\lambda} \rightarrow 0 .
$$

Here we set $u_{\lambda(0)}=0$. Using Theorem 4.1, we have

$$
\operatorname{dim}_{\mathbb{C}} \operatorname{Ext}^{1}\left(u_{\lambda}, u_{\lambda(n)}\right) X_{u_{\lambda}} X_{u_{\lambda(n)}}=X_{u_{\lambda(n+1)}}+X_{u_{\lambda(n-1)}} .
$$

It is clear that $X_{u_{\lambda(n)}}$ does not depend on the choice of $\lambda \in \mathbb{P}^{1}(\mathbb{C})$. We denote it by $r_{n}$. Set $r_{0}=1$. Hence, we have

$$
r_{1}=x_{0} x_{3}-x_{1} x_{2} \quad \text { and } \quad r_{n+1}=r_{1} r_{n}-r_{n-1},
$$

which are elements of the basis called 'dual semicanonical canonical basis' in [5] and 33. For $n=2$, it is known that $\operatorname{dim}_{\mathbb{C}} \operatorname{Ext}_{\mathbb{C} Q}^{1}\left(u_{\lambda(2)}, u_{\lambda(2)}\right)=2$. The corresponding two linearly independent extensions are as follows:

$$
0 \rightarrow u_{\lambda(2)} \rightarrow u_{\lambda(4)} \rightarrow u_{\lambda(2)} \rightarrow 0
$$

and

$$
0 \rightarrow u_{\lambda(2)} \rightarrow u_{\lambda(1)} \oplus u_{\lambda(3)} \rightarrow u_{\lambda(2)} \rightarrow 0 .
$$


The latter is the Auslander-Reiten sequence. By using Theorem 4.1, we have

$$
\operatorname{dim}_{\mathbb{C}} \operatorname{Ext}^{1}\left(u_{\lambda(2)}, u_{\lambda(2)}\right) X_{u_{\lambda(2)}} X_{u_{\lambda(2)}}=X_{u_{\lambda(4)}}+X_{u_{\lambda(1)}} X_{u_{\lambda(3)}}+X_{u_{\lambda(1)}}^{2}+1
$$

Hence, we have

$$
2 r_{2}^{2}=r_{4}+r_{1} r_{3}+r_{1}^{2}+1
$$

However, equation (4.5) tells us that $r_{1}^{2}=r_{2}+1$ and $r_{4}+r_{2}=r_{1} r_{3}$. Therefore, we have $r_{2}^{2}=r_{1} r_{3}+1$. This agrees with Proposition 4.4

\section{ACKNOWLEDGEMEnTS}

The author thanks the referee for many helpful comments. He is also grateful to Dr. Xueqing Chen and Professor Bin Zhu for interesting discussions. Finally, the author would like to thank the Max-Planck Institute for Mathematics in Bonn for a three-month research stay in 2008.

\section{REFERENCES}

1. A. Buan, R. Marsh, M. Reineke, I. Reiten, G. Todorov, Tilting theory and cluster combinatorics, Adv. Math. 204 (2006), 572-618. MR2249625 (2007f:16033)

2. P. Caldero, F. Chapoton, Cluster algebras as Hall algebras of quiver representations, Comm. Math. Helv. 81 (2006), 595-616. MR.2250855 (2008b:16015)

3. P. Caldero, B. Keller, From triangulated categories to cluster algebras, Inv. Math. 172 (2008), 169-211. MR2385670

4. P. Caldero, B. Keller, From triangulated categories to cluster algebras II, Ann. Sci. École Norm. Sup. 39 (4) (2006), no. 6, 983-1009. MR2316979 (2008m:16031)

5. P. Caldero, A. Zelevinsky, Laurent expansions in cluster algebras via quiver representations, Mosc. Math. J. 6 (2006), no. 3, 411-429. MR2274858 (2008j:16045)

6. A. Dimca, Sheaves in topology. Universitext. Springer-Verlag, Berlin, 2004. MR2050072 (2005j:55002)

7. G. Dupont, Cluster multiplication in stable tubes via generalized Chebyshev polynomials, arXiv: math/0801.3964.

8. M. Ding, J. Xiao and F. Xu, Realizing Enveloping Algebras via Varieties of Modules, arXiv: math/0604560.

9. S. Fomin, A. Zelevinsky, Cluster algebras. I. Foundations, J. Amer. Math. Soc. 15 (2002), no. 2, 497-529. MR 1887642 (2003f:16050)

10. P. Gabriel, Auslander-Reiten sequences and representation-finite algebras, Lecture Notes in Math. 831 (1980), 1-71. MR607140 (82i:16030)

11. J. A. Green, Hall algebras, hereditary algebras and quantum groups, Inv. Math. 120 (1995), 361-377. MR1329046 (96c:16016)

12. C. Geiss, B. Leclerc, J. Schröer, Semicanonical bases and preprojective algebras II: A multiplication formula, Compositio Math. 143 (2007), 1313-1334. MR 2360317

13. A. Hubery, From triangulated categories to Lie algebras: A theorem of Peng and Xiao, Proceedings of the Workshop on Representation Theory of Algebras and related Topics (Quertaro, 2004), editors J. De la Peña and R. Bautista.

14. A. Hubery, Acyclic cluster algebras via Ringel-Hall algebras, preprint.

15. A. Hubery, Hall Polynomials for Affine Quivers, preprint.

16. D. Joyce, Constructible functions on Artin stacks, J. London Math. Soc. 74 (2006), 583-606. MR 2286434 (2008b:14001)

17. B. Keller, On triangulated orbit categories, Documenta Mathematica, 10 (2005) 551-581. MR2184464 (2007c:18006)

18. G. Lusztig Quivers, perverse sheaves, and quantized enveloping algebras, J. Amer. Math. Soc. 4(2) (1991), 365-421. MR1088333 (91m:17018)

19. R. D. MacPherson, Chern classes for singular algebraic varieties, Ann. Math. 100 (1974), 423-432. MR0361141 (50:13587)

20. R. Marsh, M. Reineke, A. Zelevinsky, Generalized associahedra via quiver representations, Trans. Amer. Math. Soc. 355(1) (2003), 4171-4186. MR1990581|(2004g:52014) 
21. Y. Palu, Cluster characters for 2-Calabi-Yau triangulated categories, arXiv:math/0703540v1, to appear in Ann. Fourier.

22. N. J. Richmond, A stratification for varieties of modules, Bull. London Math. Soc. 33 (2001), no. 5, 565-577. MR.1844554 (2002d:16017)

23. Ch. Riedtmann, Lie algebras generated by indecomposables, J. Algebra 170 (1994), 526-546. MR.1302854 (96e:16013)

24. C. M. Ringel, Hall algebras and quantum groups, Inv. Math. 101 (1990), 583-592. MR1062796 (91i:16024)

25. C. M. Ringel, Green's theorem on Hall algebras, Representation theory of algebras and related topics (Mexico City, 1994), 185-245, CMS Conf. Proc. 19, Amer. Math. Soc., Providence, RI, 1996. MR 1388564 (97h:16014)

26. C. M. Ringel, The preprojective algebra of a quiver. In: Algebras and Modules, II. CMS. Proc. 24 (1998), 467-480. MR1648647 (99i:16031)

27. O. Schiffmann, Canonical bases via moduli spaces of sheaves on curves, Inv. Math. 165 (2006), 453-524. MR2242625 (2008b:17023)

28. J. P. Serre, Espaces fibrés algébriques, Seminaire C. Chevalley 1958, 1-36.

29. B. Toën, Derived Hall algebras, Duke Math. J. 135 (2006), no. 3, 587-615. MR2272977 (2007h:18021)

30. J. Xiao, F. Xu, Hall algebras associated to triangulated categories, Duke Math. J. 143 (2008), no. 2, 357-373. MR2420510

31. J. Xiao, F. Xu, Green's formula with $\mathbb{C}^{*}$-action and Caldero-Keller's formula for cluster algebras, to appear in Prog. Math.

32. J. Xiao, F. Xu, G. Zhang, Derived categories and Lie algebras, arXiv:math/0604564.

33. A. Zelevinsky, Semicanonical basis generators of the cluster algebra of type $A_{1}^{(1)}$, Electron. J. Combin. 14 (2007), no. 1, Note 4, 5 pp. (electronic). MR2285807 (2008j:05375)

Department of Mathematical Sciences, Tsinghua University, Beijing 100084, People's Republic of China

E-mail address: fanxu@mail.tsinghua.edu.cn 